Pacific Journal of Mathematics

ASYMPTOTIC BEHAVIOR OF A PERTURBED NEUTRAL
FUNCTIONAL-DIFFERENTIAL EQUATION RELATED TO THE
SOLUTION OF THE UNPERTURBED LINEAR SYSTEM 


\section{ASYMPTOTIC BEHAVIOR OF A PERTURBED NEUTRAL FUNCTIONAL DIFFERENTIAL EQUATION RELATED TO THE SOLUTION OF THE UNPERTURBED LINEAR SYSTEM}

\section{A. F. IzE AND A. VENTURA}

In this paper we consider the problem of the relative asymptotic equivalence of the solutions of the systems

$$
\frac{d}{d t} D y_{t}=L\left(y_{t}\right)
$$

and

$$
\frac{d}{d t}\left[D x_{t}-G\left(t, x_{t}\right)\right]=L\left(x_{t}\right)+f\left(t, x_{t}\right),
$$

where (1) is a linear system of neutral functional differential equations. The main theorem gives conditions under which the following result is verified. Given a solution $y_{t}$ of (1) there exists a solution $x_{t}$ of (2) such that

$$
\lim \frac{\left\|x_{t}-y_{t}\right\|}{\left\|y_{t}\right\|}=0
$$

The converse of this result, namely given a solution $x_{t}$ of (2) there is a solution $y_{t}$ of (1) such that $(*)$ is satisfied is partially proved. A counterexample is given to show that the converse result is not true in general.

0. Introduction. The study of asymptotic behavior of differential equations is very important to the understanding of the qualitative behavior of the solutions of an ordinary differential equation. Several mathematicians, including N. Levinson, H. Weil, P. Hartman, R. Bellman, K. Cooke, J. Hale, L. Cesari, and others, have done a great deal of work in this area. The theory of functional differential equations is relatively new, having evolved mainly in the last twenty years, and not many papers have appeared on asymptotic behavior of functional differential equations. One early paper was published by Bellman and Cooke in 1959 [1], followed by several others that consider a nonlinear delay equation as a perturbation of an ordinary differential equation; see, for example, Cooke [3]. Although this point of view is important, in some cases a better approach is to consider a nonlinear functional differential equation because the linearized equation is still a linear functional differential equation, and because the difficulties involved in the solution of the problem spring 
from the fact that the space of the solutions of the linear system is now an infinite-dimensional space. The first paper in this direction was published by J. Hale [7]. It is a generalization of Bellman's and Cooke's paper. A further generalization by Izé and Molfetta [9] appeared later for a more general class of neutral functional differential equations. We should point out that for functional differential equations of the neutral type the methods used to study asymptotic behavior are in some sense more effective than the classical Lyapunov second method. When we use the classical Lyapunov theorems for neutral equations we must always assume the operator $D$ is uniformly stable.

In this paper we consider the problem of the relative asymptotic equivalence of the solutions of the systems

$$
\begin{gathered}
\frac{d}{d t} D y_{t}=L\left(y_{t}\right), \\
\frac{d}{d t}\left[D x_{t}-G\left(t, x_{t}\right)\right]=L\left(y_{t}\right)+f\left(t, x_{t}\right),
\end{gathered}
$$

where (L) is a linear autonomous system of functional differential equations.

Let $a_{D}$ be the order the strongly continuous semigroup $\left\{T_{D}(t-\sigma), t\right.$ $\geq 0\}$ associated with the solutions of the difference equation $(d / d t) D x_{t}$ $=0$. If $\beta>a_{D}$ and $y_{t}$ is a solution of (L) such that $\left\|y_{t}\right\| / \exp \beta t$ does not go to zero, there exists a nonnegative integer $l$ and a real number $\alpha$ uniquely determined such that

$$
0<\lim _{t \rightarrow \infty}\left\|y_{t}\right\| / t^{t} e^{\alpha t} \leq \varlimsup_{t \rightarrow \infty}\left\|y_{t}\right\| t^{l} e^{\alpha t}<\infty .
$$

If the condition above is satisfied we say $\left\|y_{t}\right\| \sim t^{l} e^{\alpha t}$. Let $y(t)$ be a solution of (L) such that $\left\|y_{t}\right\| \sim t^{l} e^{\alpha t}$ and let $S$ be the vector subspace of $C$ defined by

$$
S=\left\{\phi \in C \mid \lim _{t \rightarrow \infty}\|T(t) \phi\| /\left\|y_{t}\right\|=0\right\} .
$$

In $\$ 2$ conditions are given on $f$ and $G$ under which there exists a subset $Y_{S}$ of $C$ and a real number $\sigma>0$ such that for every $\phi \in Y+Y_{S}$ there exists a solution $x(t)$ of $(\mathrm{P})$ such that $x_{\sigma}=\phi$ and

$$
\lim \left\|y_{t}-x_{t}\right\| /\left\|y_{t}\right\|=0 \text {. }
$$

Furthermore, $Y_{S}$ is homeomorphic to $S$.

Although the converse of this result, namely, given a solution $x_{t}$ of $(\mathrm{P})$ there exists a solution $y_{t}$ of $(\mathrm{L})$ such that $(*)$ is satisfied is true for ordinary 
differential equations, a simple counterexample given by J. Hale shows that it is not true in general for delay equations. However if the Lyapunov number of the solutions of $(\mathrm{L})$ are finite, the converse result is true and a partial converse theorem is proved.

We should remark that in the proof of Theorem 2.3 we do not assume the operator $D$ is uniformly stable. Thus it can be applied even to perturbations of linear equations that have a weird behavior such as those given by Gromova and Zverkin [4] and Brumley [2].

1. Preliminaries. Let $r>0$ be a given real number, $R=(-\infty, \infty)$, $E^{n}=R^{n}$ or $C^{n}$ a complex $n$-dimensional linear vector space with norm $|\cdot|$ and $C\left([a, b], E^{n}\right)$ the Banach space of continuous functions mapping the interval $[a, b]$ to $E^{n}$ with the topology of uniform convergence of compact sets. If $[a, b]=[-r, 0]$ then $C=C\left([-r, 0], E^{n}\right)$, and the norm in $C$ will be given by

$$
\|\phi\|=\sup _{-r \leq \theta \leq 0}|\phi(\theta)| .
$$

If $\sigma \in R, A \geq 0$ and $x \in C\left([\sigma-r, \sigma+A], E^{n}\right)$, then for every $t \in$ $[\sigma, \sigma+A]$ we let $x_{t} \in C$ be defined by $x_{t}(\theta)=x(t+\sigma),-r \leq \theta \leq 0$.

If $\Omega \subset R \times C$ is open and if $D, f: \Omega \rightarrow R^{n}$ are continuous functions we say that the relation

$$
\frac{d}{d t} D\left(t, x_{t}\right)=f\left(t, x_{t}\right)
$$

is a functional differential equation. A function $x$ is said to be a solution of (1.1) if there are $\sigma \in R$ and $A>0$ such that $x \in C\left([\sigma-r, \sigma+A) E^{n}\right)$, $\left(t, x_{t}\right) \in \Omega, t \in[\sigma, \sigma+A)$ and $x$ satisfies (1.1) on $(\sigma, \sigma+A)$. For a given $\sigma \in R, \phi \in C,(\sigma, \phi) \in \Omega$, we say that $x(\sigma, \phi)$ is a solution of (1.1) with initial value $(\sigma, \phi)$ or a solution of (1.1) through $(\sigma, \phi)$ if there is an $A>0$ such that $x(\sigma, \phi)$ is a solution of $(1.1)$ on $[\sigma-r, \sigma+A)$ and $x_{\sigma}(\sigma, \phi)=\phi$.

Let $X, Y$ be Banach spaces, $\mathcal{L}(X, Y)$ the Banach space of bounded linear mappings from $X$ into $Y$. If $L \in \mathcal{L}\left(C, E^{n}\right)$, then the Riesz representation theorem implies there is an $n \times n$ matrix function $\eta$ on $[-r, 0]$ of bounded variation such that

$$
L \phi=\int_{-r}^{0}[d \eta(\theta)] \phi(\theta) .
$$

For any such $\eta$ we always understand that we have extended the definition to $R$ so $\eta(\theta)=\eta(-r)$ for $0 \leq-r, \eta(\theta)=\eta(0)$ for $\theta \geq 0$. 
Let $\Lambda$ be an open subset of a metric space. We say $L: \Lambda \rightarrow \mathcal{L}\left(C, R^{n}\right)$ has smoothness on the measure if for any real $\beta$ there is a scalar function $\gamma(\lambda, s)$ continuous for $\lambda \in \Lambda, s \in R, \gamma(\lambda, 0)=0$ such that if

$$
L(\lambda) \phi=\int_{-r}^{0}[d \eta(\lambda, \theta)] \phi(\theta), \quad \lambda \in \Lambda, 0<s,
$$

then

$$
\left|\lim _{h \rightarrow 0^{+}} \int_{\beta+h}^{\beta+s}+\int_{\beta-s}^{\beta-h}[d \eta(\lambda, \theta)] \phi(\theta)\right| \leq \gamma(\lambda, s)\|\phi\| .
$$

If $\beta \in R$ and the matrix $A(\lambda ; \beta, L)=\eta\left(\lambda, \beta^{+}\right)-\eta\left(\lambda, \beta^{-}\right)$is nonsingular on $\lambda=\lambda_{0}$, we say $L(\lambda)$ is atomic at $\beta$ at $\lambda_{0}$. If $A(\lambda ; \beta, L)$ is nonsingular on a set $K \subset \Lambda$ we say $L(\lambda)$ is atomic at $\beta$ on $K$.

Let $\Lambda=\Omega \subset R \times C$ and $L \in C\left(\Omega, \mathcal{L}\left(C, R^{n}\right)\right)$.

If $D: \Omega \rightarrow R^{n}$ has a continuous first derivative with respect to $\phi$, then Lemma 5.1 of Hale [5], p. 50, implies $D_{\phi}$ has smoothness on the measure.

Definition 1.1. Suppose $\Omega \subset R \times C$ is an open set and $(t, \phi) \in \Omega$. A function $D: \Omega \rightarrow R^{n}$ (not necessarily linear) is said to be atomic at $\beta$ on $\Omega$ if $D$ is continuous together with its first and second Fréchet derivatives with respect to $\phi$, and $D_{\phi}$, the derivative with respect to $\phi$, is atomic at $\beta$ on $\Omega$.

System (1.1) is called a functional differential equation of neutral type if $D$ is atomic at zero.

We also assume the existence, uniqueness, and continuous dependence with respect to initial condition of the solutions of (1.1).

Assume $\Omega \subset C$ is open, and $D: \Omega \rightarrow R^{n}$ is a continuous linear operator given by

$$
D_{\phi}=\phi(0)-g(\phi) \text { with } g(\phi)=\int_{-r}^{0}[d \mu(\theta)] \phi(\theta),
$$

where $\mu(\theta),-r \leq \theta \leq 0$, is an $n \times n$ matrix whose elements are of bounded variation and do not have singular parts, that is,

$$
\int_{-r}^{0}[d \mu(\theta)] \phi(\theta)=\sum_{k=1}^{\infty} A_{k} \phi\left(-w_{k}\right)+\int_{-r}^{0} A(\theta) \phi(\theta) d \theta,
$$

where

$$
0<w_{k} \leq r \text { and } \sum_{k=1}^{\infty}\left|A_{k}\right|+\int_{-r}^{0}|A(\theta)| d \theta<\infty
$$


In [5] page 304 we found the following.

Definition 1.2. If $G: C \rightarrow R^{n}$ is continuous we say $G(\phi)$ is independent of $\phi(0)$ if there is an $\varepsilon \in[-r, 0)$ such that $G(\phi)$ depends only on the values $\phi(\theta)$ of the function $\phi$ for $\theta \in[-r, \varepsilon)$. The systems

$$
\frac{d}{d t} D x_{t}=L x_{t}
$$

and

$$
\frac{d}{d t}\left[D x_{t}-G\left(t, x_{t}\right)\right]=L x_{t}+f\left(t, x_{t}\right)
$$

are our main concern, where $D$ satisfies (1.4), $L$ satisfies (1.2), $D x_{t}-$ $G\left(t, x_{t}\right)$ is atomic at zero and $G(t, \phi)$ does not depend on $\phi(0), \phi=x_{\sigma}$.

If $\phi \in C$ and $x_{t}(\sigma, \phi)$ is the unique solution of (1.6) we define the operator $T(t-\sigma): C \rightarrow C, t \geq \sigma$, by the relation

$$
x_{t}(\sigma, \phi)=T(t-\sigma), \quad T(0)=I .
$$

$\{T(t-\sigma)\}_{t \in[\sigma, \infty)}$ is a family of strongly continuous semigroups from $C$ into itself for all $t \geq \sigma$.

In [5] Theorem 10.1, p. 307, is proved that:

THEOREM 1.3. (i) The infinitesimal generator $A$ of a semigroup $T(t-\sigma)$, $t \geq \sigma$, of (1.6) has domain $\mathscr{D}(A)$ and range $\Re(A)$, respectively, given by

$$
\mathscr{D}(A)=\{\phi \in C: \dot{\phi} \in C, D \dot{\phi}=L \dot{\phi}\}, \quad A \dot{\phi}=\dot{\phi} .
$$

(ii) The spectrum $\sigma(A)$ coincides with the point spectrum (eigenvalues), and $\lambda \in \sigma(A)$ if and only if $\lambda$ satisfies the characteristic equation

$$
\operatorname{det} \Delta(\lambda)=0, \quad \Delta(\lambda)=\lambda D\left(e^{\lambda^{*}} I\right)-L\left(e^{\lambda^{\bullet}} I\right) .
$$

(iii) The roots of characteristic equations have real parts bounded above, and if $\lambda \in \sigma(A)$ then the generalized eigenspace $\mathfrak{K}_{\lambda}(A)$ is finite dimensional and there is an integer $k=k(\lambda)$ such that

$$
\Re_{\lambda}(A)=\Re(A-\lambda I)^{k} \text { and } C=\Re(A-\lambda I)^{k} \oplus \Re(A-\lambda I)^{k} .
$$

(iv) Suppose $\Lambda$ is a finite set $\left\{\lambda_{1}, \lambda_{2}, \ldots, \lambda_{p}\right\}$ of elements of $\sigma(A)$, and $\Phi_{\Lambda}=\left(\Phi_{\lambda_{1}}, \ldots, \Phi_{\lambda_{p}}\right), B_{\Lambda}=\left(B_{\lambda_{1}}, \ldots, B_{\lambda_{p}}\right)$, where $\Phi_{\lambda_{1}}$ is a basis for the generalized eigenspace of $\lambda_{j}$, and $B_{\lambda_{1}}$ is the matrix defined by $A \Phi_{\lambda_{j}}=\Phi_{\lambda_{j}}$, $j=1,2, \ldots, p$. Then the only eigenvalue of $B_{\lambda}, i s \lambda$, and, for any eigenvector a of the same dimension as $\Phi_{\Lambda}$, the solution $T(t-\sigma) \Phi_{\Lambda}$ a of (1.4) with initial 
value $\Phi_{\Lambda}$ a at $t=\sigma$ may be defined on $(-\infty, \infty)$ by the relation

$$
T(t-\sigma) \Phi_{\Lambda} a=\Phi_{\Lambda} e^{B_{\Lambda}(t-\sigma)} a \Phi_{\Lambda}(\theta)=\Phi_{\Lambda}(0) e^{B_{\Lambda} \theta}, \quad-r \leq \theta \leq 0 .
$$

Furthermore, there exists a subspace $Q_{\Lambda}$ of $C$ such that $T(t-\sigma) Q_{\Lambda} \subset$ $Q_{\Lambda}, t \geq \sigma$, and $C=P_{\Lambda} \oplus Q_{\Lambda}$, where

$$
P_{\Lambda}=\left\{\phi \in C: \phi=\Phi_{\Lambda} a, \text { for some vector } a\right\} .
$$

We can give an explicit characterization of decomposition of space $C$ via the formal adjoint equation

$$
\frac{d}{d s}\left[y(s)-\int_{-r}^{0} y(s-\theta) d \mu(\theta)\right]=-\int_{-r}^{0} y(s-\theta) d \eta(\theta)
$$

where $y$ is an $n$-dimensional row vector. If $C^{*}=C\left([0, r], E^{n^{*}}\right)$, where $E^{n^{*}}$ is the $n$-dimensional space of row vectors, then, for any $\phi \in C$ the bilinear forms

$$
\begin{aligned}
(\alpha, \phi)= & \alpha(0) \phi(0)+\int_{-r}^{0}\left[\frac{d}{d u} \int_{0}^{u} \alpha(s-u) d \mu(\theta) \phi(s) d s\right]_{u=\theta} \\
& -\int_{-r}^{0} \int_{0}^{\theta} \alpha(s-\theta) d \eta(\theta) \phi(s) d s
\end{aligned}
$$

are defined for $\alpha \in C^{*}=C\left([0, r], E^{n^{*}}\right), \dot{\alpha} \in C^{*}, \phi \in C$.

The following theorem is proved in [5], p. 309.

THEOREM 1.4. If $\Lambda=\left\{\lambda_{1}, \ldots, \lambda_{p}\right\}$ is a finite set of elements of $\sigma(A)$ and $P_{\Lambda}$ is the linear extension of $\Re_{\lambda_{1}}(A), j=1, \ldots, p$, with basis $\phi_{\Lambda}$, and $p_{\Lambda}^{*}$ is the linear extension of the correspondent generalized eigenspace of the formal adjoint equation with basis $\Psi_{\Lambda}$, then one can choose $\Psi_{\Lambda}$ so that $\left(\Psi_{\Lambda}, \Phi_{\Lambda}\right)=I$ is the identity and

$$
\begin{aligned}
C & =P_{\Lambda} \oplus Q_{\Lambda}, \\
P_{\Lambda} & =\left\{\phi \in C: \phi=\phi_{\Lambda} a, \text { for some vector } a\right\}, \\
Q_{\Lambda} & =\{\phi \in C:(\Psi, \phi)=0\} .
\end{aligned}
$$

In the condition of Theorem 1.4 we say that the space $C$ is decomposed by $\Lambda$ and therefore if $\phi \in C$, we have $\phi=\phi^{P_{\Lambda}}+\phi^{Q_{\Lambda}}$, where $\phi^{P_{\Lambda}}=\Phi a, a=$ $\left(\Psi_{A}, \phi\right)$ and $\phi^{Q_{\Lambda}}=\phi-\phi^{P_{\Lambda}}$.

Furthermore, if $C$ is decomposed by $\Lambda$, then $\sigma\left(T(t-\sigma) \mid P_{\Lambda}\right)=$ $\sigma\left(e^{B_{\Lambda}(t-\sigma)}\right)$, where $A \Phi_{\Lambda}=\Phi_{\Lambda} B_{\Lambda}$ and $A \Psi_{\Lambda}=B_{\Lambda} \Psi_{\Lambda}$. 
1.2. Variation of constant formula. Consider $\left\{T_{D}(t-\sigma), t \geq \sigma\right\}$, the strongly continuous semigroup of linear mappings associated with the solution of the difference equation $(d / d t) D x_{t}=0$.

Definition 1.5. The order $a_{D}$ of the semigroup is defined by

$$
a_{D}=\inf \left\{a \in R: \exists K=K(a) \text { with }\left\|T_{D}(t-\sigma)\right\| \leq K e^{a(t-\sigma)}, t \geq \sigma\right\} .
$$

In [5] we can find some results that can be stated as

THEOREM 1.6. If $D$ and $L$ satisfy (1.6), the matrix $\mu$ has nonsingular part, and $a>a_{D}$ is fixed, then the set $\Lambda_{a}=\{\lambda \in \sigma(A): \operatorname{Re} \lambda \geq a\}$ is finite and the space $C$ can be decomposed by $\Lambda_{a}$ as $C=P \oplus Q$, where $P$ and $Q$ are invariant subspaces under $T(t-\sigma)$ and $A$, and the space $P$ is finite dimensional and corresponds to the initial data of all those solutions of (1.6) which are of the form $p(t) \exp (\lambda t)$, where $p(t)$ is a polynomial in $t$ and $\lambda \in \Lambda_{a}$.

If $x_{t}(\sigma, \phi)$ is a solution of (1.6), then, according to Theorems 1.3 and 1.4, we may write $x_{t}=x_{t}^{P}+X_{t}^{Q}$.

Now let $X(t), t \geq \sigma$, be the $n \times n$ matrix function defined for all $t \in[0, \infty)$ of bounded variation in $t$ and continuous in $t$ from the right such that

$$
\begin{gathered}
D\left(X_{t-\sigma}\right)=I+\int_{\sigma}^{t} L\left(X_{s-\sigma}\right) d s, \quad t \geq \sigma, \\
X_{0}(\theta)= \begin{cases}0, & -r \leq \theta<0, \\
I, & \theta=0 .\end{cases}
\end{gathered}
$$

So according to [5], page 302, a solution of (1.7) with initial value $\phi$ in $\sigma$ satisfies the variation of constants formula

$$
\begin{aligned}
x_{t}-X_{0} G\left(t, x_{t}\right)= & T(t-\sigma)\left[\phi-X_{0} G(0, \phi)\right] \\
+\int_{\sigma}^{t}\left\{\left[-d s T(t-s) X_{0}\right] G\left(s, x_{s}\right)\right. & \\
& \left.\quad+T(t-s) X_{0} f\left(s, x_{s}\right) d s\right\} \quad t \geq \sigma,
\end{aligned}
$$

where $T(t-\sigma)\left[\phi-X_{0} G(0, \phi)\right]$ is defined as $T(t-\sigma) \phi-X_{t} G(0, \phi)$ and

$$
X(t-\sigma) X_{0}=X_{t-\sigma} .
$$


The integrals in (1.14) are evaluated at each $\theta \in[-r, 0]$ as ordinary integrals in $E^{n}$. Also, if $C$ is decomposed by $\Lambda$ as $C=P \oplus Q$, then (1.14) is equivalent to

$$
\begin{aligned}
x_{t}^{P}- & X_{0}^{P} G\left(t, x_{t}\right) \\
= & T(t-\sigma)\left[\phi^{P}-X_{0}^{P} G(0, \phi)\right] \\
\quad & \quad \int_{\sigma}^{t}\left\{\left[-d s T(t-s) X_{0}^{P}\right] G\left(s, x_{s}\right)+T(t-s) X_{0}^{P} f\left(s, x_{s}\right) d s\right\} \\
X_{t}^{Q}-X_{0}^{Q} G\left(t, x_{t}\right) & \\
= & T(t-\sigma)\left[\phi^{Q}-X_{0}^{Q} G(0, \phi)\right] \\
\quad+ & \int_{\sigma}^{t}\left\{\left[-d s T(t-s) X_{0}^{Q}\right] G\left(s, x_{s}\right)+T(t-s) X_{0}^{Q} f\left(s, x_{s}\right) d s\right\}
\end{aligned}
$$

where the superscripts $P$ and $Q$ designate the projection of the corresponding function onto the subspaces $P$ and $Q$, respectively.

However, we must observe that everything is clear in (1.16) except for the meaning of the projections $X_{0}^{P}, X_{0}^{Q}$ since $X_{0}$ is not continuous. Projection operators taking $C$ onto $P$ and $C$ are easily determined by means of the adjoint differential equation (1.10) and the bilinear form (1.11). One can show that $\left(\Psi, X_{0}\right)$ is well defined and $\left(\Psi, X_{0}\right)=\Psi(0)$.

Therefore if we put

$$
X_{0}^{P}=\Phi \Psi(0), \quad X_{0}^{Q}=X_{0}-X_{0}^{P},
$$

the quantities in (1.16) are well defined.

Also in [8], Henry has given some exponential estimates for the solutions of (1.6).

THEOREM 1.7. If $\alpha>a_{D}$ and $\Lambda=\{\lambda \in \sigma(A): \operatorname{Re} \lambda>\alpha$, $\operatorname{det} \Delta(\lambda)=$ $0\}$, then $C$ is decomposed by $\Lambda$ as $C=P \oplus Q$ and there are positive constants $M_{1}, M_{2}, M_{3}, M_{4}$ and $\varepsilon$ such that for $\sigma \geq 0$,

$$
\begin{aligned}
& \left\|T(t-\sigma) \phi^{Q}\right\| \leq M_{1} e^{(\alpha-\varepsilon)(t-\sigma)}\left\|\phi^{Q}\right\|, \quad t \geq \sigma, \quad \phi^{Q} \in Q, \\
& \left\|T(t-\sigma) \phi^{P}\right\| \leq M_{2} e^{(\alpha+\varepsilon)(t-\sigma)}\left\|\phi^{P}\right\|, \quad t \geq \sigma, \quad \phi^{P} \in P,
\end{aligned}
$$

and

$$
\begin{gathered}
\left\|T(t-\sigma) X_{0}^{Q}\right\| \leq M_{3} e^{(\alpha-\varepsilon)(t-\sigma)}, \quad t \geq \sigma, \\
\left\|T(t-\sigma) X_{0}^{P}\right\| \leq M_{4} e^{(\alpha+\varepsilon)(t-\sigma)}, \quad t \leq \sigma, \\
\int^{\infty}\left\|d_{s} T(s) X_{0}^{Q}\right\| e^{-(\alpha-\varepsilon) s} \leq M_{3}, \\
\int^{\infty}\left\|d_{s} T(s) X_{0}^{P}\right\| e^{-(\alpha+\varepsilon) s} \leq M_{4} .
\end{gathered}
$$


Relation (1.14) suggest the possibility of introducing a new variable for the expression on the left-hand side. However, some care must be exercised because the new variable would not be a continuous function on $[-r, 0]$. See [5] page 302.

The variation of constants formula (1.14) for the solutions of (1.7) suggests the change of variables

$$
z_{t}=x_{t}-X_{0} G\left(t, x_{t}\right)
$$

to obtain a new equation for $z_{t}$ in $P C$, where $P C$ is the space of functions $\phi:[-r, 0] \rightarrow R^{n}$ which are uniformly continuous on $[-r, 0)$ and for which there exists $\phi\left(0^{-}\right)$. See [5] page 302.

The transformation (1.21) is a well-defined transformation from $C$ to $P C$, since $G(t, \phi)$ is independent of $\phi(0)$. Therefore, $G\left(t, x_{t}\right)=G\left(t, z_{t}\right)$ for $-r \leq \theta<0$.

Thus if

$$
\text { (1.22) } z_{t}=x_{t}-X_{0} G\left(t, x_{t}\right), \quad x_{t}=z_{t}+X_{0} G\left(t, x_{t}\right) \stackrel{\text { def }}{=} H\left(t, z_{t}\right) \text {, }
$$

(1.14) becomes

$$
\begin{aligned}
z_{t}=T(t-\sigma) z_{\sigma}+\int_{\sigma}^{t}\{[-d s & \left.T(t-s) X_{0}\right] G\left(s, z_{s}\right) \\
& \left.+T(t-s) X_{0} F\left(s, H\left(s, z_{s}\right)\right) d s\right\}
\end{aligned}
$$

Let $\Phi, \Psi$ be the matrices defined by the composition $C=P \oplus Q$, $(\Psi, \Phi)=I$ and let $B$ the $p \times p$ matrix such that $T(t) \Phi=\Phi \exp (B t)$, $t \in(-\infty, \infty)$. The spectrum of $B$ is $\Lambda$. For any $\phi \in P C$ one can define $(\psi, \phi)$ and, therefore, it is meaningful to put

$$
\phi^{P}=\Phi(\psi, \phi), \quad \phi^{Q}=\phi-\phi^{P}, \quad \phi \in P C .
$$

One can show that $\left(\psi, X_{0}\right)$ is well defined and $\left(\Psi, X_{0}\right)=\Psi(0)$. Therefore, if we put

$$
X_{0}^{P}=\Phi \Psi(0), \quad X_{0}^{Q}=X_{0}-X_{0}^{P},
$$

the quantities in (1.14) are well defined. If we apply appropriately relations (1.22), we can split (1.23) as

$$
\begin{array}{r}
z_{t}^{P}=T(t-\sigma) z_{\sigma}^{P}+\int_{\sigma}^{t}\left\{\left[-d s T(t-s) X_{0}^{P}\right] G\left(s, z_{s}\right)\right. \\
\left.+T(t-s) X_{0}^{P} F\left(s, H\left(s, z_{s}\right)\right) d s\right\} \\
z_{t}^{Q}=T(t-\sigma) z_{\sigma}^{Q}+\int_{\sigma}^{t}\left\{\left[-d s T(t-s) X_{0}^{Q}\right] G\left(s, z_{s}\right)\right. \\
\left.+T(t-s) X_{0}^{Q} F\left(s, H\left(s, z_{s}\right)\right) d s\right\}
\end{array}
$$


Definition 1.8. The operator $D$ is said to be uniformly stable if there exist constants $K \geq 1$ and $\alpha>0$ such that

$$
\left\|T^{D}(t) \phi\right\| \leq K e^{-\alpha t}\|\phi\|, \quad \phi \in C^{0}, \quad t \geq 0 .
$$

It is shown in [5] that $D$ uniformly stable implies there exists an $n \times n$ matrix function $B(t)$ defined and of bounded variation on $[-r, \infty)$, continuous from the left, $B(t)=0,-r \leq t \leq 0$, and a constant $M>0$ such that

$$
\left\|T^{D}(t) \phi\right\| \leq M\|\phi\|, \quad t \geq 0, \quad \phi \in C, \quad \sup _{t \geq-r}|B(t)| \leq M .
$$

THEOREM 1.9. The following conditions are equivalent:

(i) $D$ is uniformly stable;

(ii) $a_{D}<0$, where $a_{D}$ is the order of the semigroup $T^{0}(t)$;

(iii) there are constants $a, b>0$ such that for any $h \in C\left([0, \infty), R^{n}\right)$, any solution $y$ of the inhomogeneous equation $D y_{t}=h(t), t \geq 0$, satisfies

$$
\left\|y_{t}\right\| \leq b e^{-a t}\left\|y_{0}\right\|+b \sup _{0 \leq u \leq t}|h(u)|, \quad t \geq 0 ;
$$

(iv) if $D \phi=\phi(0)-\int_{-r}^{0}[d \mu(\theta)] \phi(\theta)$, where $\mu$ satisfies conditions (1.5) of $\S 1$, there exists $\delta>0$ such that all solutions of the characteristic equation $\operatorname{det}\left[I-\int_{-r}^{0} e^{\lambda \theta} d \mu(\theta)\right]=0$ satisfy $\operatorname{Re} \lambda \leq-\delta$.

2. Main results. The lemma below gives a characterization on the asymptotic behavior of the solutions of the linear neutral differential equation

$$
\frac{d}{d t} D y=L y_{t}
$$

where $D$ and $L$ are linear.

LEMMA 2.1. If $\beta>a_{D}$ and $y_{t}$ is a solution of (2.1) such that $\left\|y_{t}\right\| /(\exp \beta t)$ does not go to zero as $t \rightarrow \infty$, there exists a nonnegative integer l and a real number $\alpha$ uniquely determined such that

$$
0<\underset{t \rightarrow \infty}{\lim }\left\|y_{t}\right\| / t^{l} e^{\alpha t} \leq \varlimsup_{t \rightarrow \infty}\left\|y_{t}\right\| / t^{l} e^{\alpha t}<\infty .
$$

Proof. If $\beta>a_{D}$ it follows from Theorem 1.7 that the space $C$ is decomposed by the set

$$
\Lambda=\{\lambda \in \sigma(A): \operatorname{Re} \lambda \geq \beta, \operatorname{det} \Delta(\lambda)=0\}
$$


as $C=P \oplus Q$, where the subspace $P$ is of finite dimension and there are positive constants $M_{1}, M_{2}$ and $\delta$ such that

$$
\begin{array}{ll}
\left\|T(t) \phi^{Q}\right\| \leq M_{2} e^{(\beta-\delta) t}\left\|\phi^{Q}\right\|, & t \geq 0, \quad \phi^{Q} \in Q, \\
\left\|T(t) \phi^{P}\right\| \leq M_{1} e^{(\beta+\delta) t}\left\|\phi^{P}\right\|, & t \leq 0, \quad \phi^{P} \in P .
\end{array}
$$

Without loss of generality we can assume $y_{t}=T(t) \phi$ for some $\phi \in C$, and since $\left\|y_{t}\right\| / e^{\beta t}$ does not tend to zero as $t \rightarrow \infty$, we have

$$
\left\|T(t) \phi^{Q}\right\| / e^{\beta t} \rightarrow 0 \quad \text { as } t \rightarrow \infty
$$

and

$$
\left\|T(t) \phi^{P}\right\| / e^{\beta t} \nrightarrow 0 \quad \text { as } t \rightarrow \infty .
$$

From (1.9) of Theorem 1.3 we have

$$
T(t) \phi^{P}=\phi e^{B t} b,
$$

where $B$ is a Jordan matrix with eigenvalues which are the elements of $\Lambda$ and have a real part greater than or equal to $\beta$.

Let

$$
z(t) \stackrel{\text { def }}{=} T(t) \phi^{P}(0)=\phi(0) e^{B t} b
$$

There are nonnegative integers $l$ and a real number $\alpha, \alpha \geq \beta$, such that

$$
0<\lim _{t \rightarrow \infty}|z(t)| / t^{l} e^{\alpha t} \leq \varlimsup_{t \rightarrow \infty}|z(t)| / t^{l} e^{\alpha t}
$$

We claim that

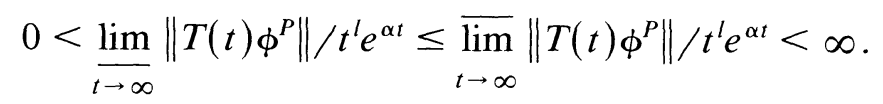

In fact from (2.2) it follows that there are positive numbers $c_{1}, c_{2}$ and $T_{1}$ such that

$$
c_{1} \leq|z(t)| / t^{t} e^{\alpha t} \leq c_{2} \text { for } t \geq T_{1}-r .
$$

But for $\theta \in[-r, 0]$,

$$
\frac{|z(t+\theta)|}{t^{\prime} e^{\alpha t}}=\frac{|z(t+\theta)|}{(t+\theta)^{\prime} e^{\alpha(t+\theta)}} \cdot e^{\alpha \theta}\left(1+\frac{\theta}{t}\right)^{\prime}>0 .
$$


It is easy to see there is $T_{2} \geq T_{1}$, large enough, such that $t \geq T_{2}$ and $\theta \in[-r, 0]$. We have

$$
\frac{|z(t+\theta)|}{t^{\prime} e^{\alpha t}} \geq \frac{c_{1}}{2} \cdot e^{\alpha \theta} \geq \frac{c_{1}}{2} \min \left\{e^{-\alpha r}, 1\right\}>0
$$

and

$$
\frac{|z(t+\theta)|}{t^{l} e^{\alpha t}} \leq c_{2} \max \left\{e^{-\alpha r}, 1\right\}<\infty .
$$

The above inequalities imply (2.3) is true.

Since $\alpha \geq \beta$ it follows that

$$
\underset{t \rightarrow \infty}{\lim _{t \rightarrow \infty}}\left\|T(t) \phi^{Q}\right\| / t^{l} e^{\alpha t}=0
$$

and then

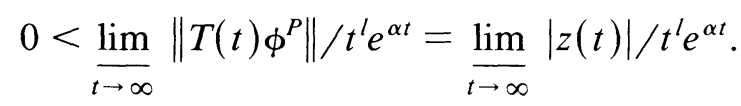

and

$$
\varlimsup_{t \rightarrow \infty}\|T(t) \phi\| / t^{l} e^{\alpha t}=\varlimsup_{t \rightarrow \infty}\left\|T(t) \phi^{P}\right\| / t^{l} e^{\alpha t}<\infty,
$$

and the proof is complete.

Notation. We say $\left\|y_{t}\right\| \sim t^{\prime} e^{\alpha t}$ if

$$
0<\lim _{t \rightarrow \infty}\left\|y_{t}\right\| / t^{l} e^{\alpha t} \leq \varlimsup_{t \rightarrow \infty}\left\|y_{t}\right\| / t^{l} e^{\alpha t}<\infty .
$$

Now consider $\beta>a_{D}$ and $y(t)$ a solution of (2.1) such that $\left\|y_{t}\right\| \sim t^{\prime} e^{\alpha t}$ with $l \in Z_{+_{+}}, \alpha \in R, \alpha \geq \beta$. Let $\Lambda=\{\lambda \in \sigma(A): \operatorname{Re} \lambda \geq \alpha$, det $\Delta(\lambda)=$ 0 . Let $P, Q$ and $B$ be as in Lemma 2.1 and let $N$ be the order of the larger block of $B$ which has in the diagonal an integer with real part equal to $\alpha$. Let

$$
P_{1}=\left\{\phi \in P: \lim _{t \rightarrow \infty}\|T(t) \phi\| / t^{l} e^{\alpha t}=0\right\}
$$

The next lemma is proved in detail in [10]. 
LEMMA 2.2. There exists a subspace $P_{2}$ of $P$ and there are projections $X^{P}: P \rightarrow P_{i}, i=1,2$, such that $P=P_{1} \oplus P_{2}, X^{P_{1}}+X^{P_{2}}=I$. Furthermore there are positive constants $M$ and $\sigma$, such that

$$
\begin{aligned}
& \left\|T(t) X^{P_{1}} T(-s) \phi^{P}\right\| \leq M t^{l-1} s^{N-l} e^{\alpha(t-s)}\left\|\phi^{P}\right\|, \quad \sigma \leq s \leq t, \\
& \left\|T(t) X^{P_{2}} T(-s) \phi^{P}\right\| \leq M t^{l} s^{N-l-1} e^{\alpha(t-s)}\left\|\phi^{P}\right\|, \quad s \geq t \geq \sigma .
\end{aligned}
$$

Now consider the systems

$$
\begin{gathered}
\frac{d}{d t} D y_{t}=L y_{t}, \\
\frac{d}{d t}\left[D x_{t}-G\left(t, x_{t}\right)\right]=L x_{t}+f\left(t, x_{t}\right),
\end{gathered}
$$

where $D, L$ are linear and $G(t, \phi)$ is independent of $\phi(0)$. (See Definition 1.2.)

Let $\beta>a_{D}$ and $y_{t}$ be a solution of (L) such that $\left\|y_{t}\right\| \sim t^{t} e^{\alpha t}$ with $\alpha \geq \beta$. Let $\Lambda=\{\lambda \in \sigma(A): \operatorname{Re} \lambda \geq \alpha\}, P, Q$ and $B$ be as in Lemma 2.1 and let $N$ be the larger order of the blocks of $B$ which have in the main diagonal integers with real part equal to $\alpha$.

The result below is the main theorem of this section.

THEOREM 2.3. Let $y(t)$ be a solution of $(\mathrm{L})$ such that $\left\|y_{t}\right\| \sim t^{l} e^{\alpha t}$. Let $S$ be the vector subspace of $C$ defined by

$$
S=\left\{\phi \in C: \lim _{t \rightarrow \infty}\|T(t) \phi\| /\left\|y_{t}\right\|=0\right\} .
$$

Assume that for $\phi, \Psi \in C$,

$$
\begin{gathered}
f(t, 0)=0, \quad|f(t, \phi)-f(t, \psi)| \leq h_{2}(t)\|\phi-\psi\|, \quad t \geq 0, \\
G(t, 0)=0, \quad|G(t, \phi)-G(t, \psi)| \leq h_{1}(t)\|\phi-\psi\|, \quad t \geq 0, \\
\int^{\infty} s^{N-1} h_{2}(s) d s<\infty, \quad \int^{\infty} s^{N-1} h_{1}(s) d s<\infty, \quad \lim _{t \rightarrow \infty} h_{1}(t)=0 .
\end{gathered}
$$

Then there is a subset $Y_{S}$ of $C$ and a real number $\sigma>0$ such that:

(a) For every $\phi \in Y_{S}$ there exists a solution $x(t)$ of $(\mathrm{P})$ such that $x_{\sigma}=\phi$ and

$$
\lim _{t \rightarrow \infty}\left\|y_{t}-x_{t}\right\| /\left\|y_{t}\right\|=0 \text {. }
$$

(b) $Y_{S}$ is homeomorphic to $S$, that is, there exists a homeomorphism $W$ : $S \rightarrow Y_{S}$ such that $W^{-1}$ is the restriction to $Y_{S}$ of a projection $X^{S}$ from $C$ onto $S$. 
Proof. Let $y(t)$ be a solution of (L) such that $\left\|y_{t}\right\| \sim t^{l} e^{\alpha t}$. Let $x=y+\bar{z}$. To find solutions $x(t)$ of $(\mathrm{P})$ satisfying (2.8) is then equivalent to finding solutions of

$$
\begin{aligned}
\frac{d}{d t}\left[D \bar{z}_{t}-G\left(t, \bar{z}_{t}\right)\right] & =L \bar{z}_{t}+f\left(t, y_{t}+\bar{z}_{t}\right) \\
& \stackrel{\text { def }}{=} L \bar{z}_{t}+F\left(t, \bar{z}_{t}\right)
\end{aligned}
$$

satisfying

$$
\lim _{t \rightarrow \infty}\left\|\bar{z}_{t}\right\| / t^{l} e^{\alpha t}=0
$$

Let $P_{1}=\left\{\phi \in P: \lim _{t \rightarrow \infty}\|T(t) \phi\| / t^{t} e^{\alpha t}=0\right\}$ and let $P_{2}, X^{P_{1}}$ and $X^{P_{2}}$ be defined as in Lemma 2.2.

Now consider the following integral equation:

$$
\begin{aligned}
z_{t}= & T(t-\sigma) \phi^{s} \\
& +\int_{\sigma}^{t}\left\{\left[-d s T(t-s) X_{0}^{Q}\right] G(s, z)+T(t-s) X_{0}^{Q} F\left(s, H\left(s, z_{s}\right)\right) d s\right\} \\
& +\int_{\sigma}^{t}\left\{\left[-d s T(t) X^{P_{1}} T(-s) X_{0}^{P}\right] G\left(s, z_{s}\right)\right. \\
& \left.+T(t) X^{P_{1}} T(-s) X_{0}^{P} F\left(s, H\left(s, z_{s}\right)\right) d s\right\} \\
& -\int_{t}^{\infty}\left\{\left[-d s T(t) X^{P_{2}} T(-s) X_{0}^{P}\right] G\left(s, z_{s}\right)\right. \\
& \left.\quad+T(t) X^{P_{2}} T(-s) X_{0}^{P} F\left(s, z_{s}\right) d s\right\}
\end{aligned}
$$

where $\phi^{S}$ is an arbitrarily fixed element of $S, X_{0}^{P}=\Phi \psi(0), X_{0}^{Q}=X_{0}-X_{0}^{P}$.

We shall show that if $z_{t}$ satisfies (2.10) then the last integral above converges. First we need to emphasize some facts. From Theorem 1.3 we know the subspace $P=\{\phi \in C: \phi=\phi a$, for some vector $a\}$ and $T(-s) \Phi a$ $=\Phi e^{-B s} a$. Thus for $a=B(\Psi, \phi)$, we have

$$
\begin{aligned}
-d s T(t) X^{P_{2}} \Phi e^{-B s}(\Psi, \phi) & =T(t) X^{P_{2}} e^{-B s} B(\Psi, \phi) d s \\
& =T(t) X^{P_{2}} T(-s) \Phi a d s
\end{aligned}
$$

with $\Phi a \in P$. 
From (1.22) we have

$$
H\left(t, z_{t}\right)=z_{t}+X_{0} G\left(t, z_{t}\right)=\bar{z}_{t} .
$$

Therefore

$$
\begin{aligned}
\left|F\left(t, H\left(t, z_{t}\right)\right)\right| & =\left|f\left(t, y_{t}+H\left(t, z_{t}\right)\right)\right| \leq h_{2}(s)\left\|y_{t}+H\left(t, z_{t}\right)\right\| \\
& \leq h_{2}(t)\left[\left\|y_{t}\right\|+\left[1+h_{1}(t)\right]\left\|z_{t}\right\|\right] .
\end{aligned}
$$

If $z_{t}$ satisfies (2.10) with $\lim _{t \rightarrow \infty} h_{1}(t)=0$, then

$$
t^{-l} e^{-\alpha t}\left[\left\|y_{t}\right\|+\left[1+h_{1}(t)\right]\left\|z_{t}\right\|\right]
$$

is bounded.

We will show that if $z_{t}$ satisfies (2.10) then the last integral of (2.11) is convergent.

In fact, from (2.5)-(2.7), we have

$$
\begin{aligned}
\| \int_{t}^{\infty}\{ & {\left[-d s T(t) X^{P_{2}} T(-s) X_{0}^{P}\right] G\left(s, z_{s}\right) } \\
& \left.+T(t) X^{P_{2}} T(-s) X_{0}^{P} F\left(s, H\left(s, z_{s}\right)\right) d s\right\} \| \\
\leq & \int_{t}^{\infty}\left\|T(t) X^{P_{2}} T(-s) \Phi a\right\|\left|G\left(s, z_{s}\right)\right| d s \\
& \quad+\int_{t}^{\infty}\left\|T(t) X^{P_{2}} T(-s) X_{0}^{P}\right\|\left|F\left(s, H\left(s, z_{s}\right)\right)\right| d s \\
\leq & M t^{l} e^{\alpha t} \int_{t}^{\infty} s^{N-1} h_{1}(s) s^{-l} e^{-\alpha s}\left\|z_{s}\right\| d s+M t^{l} e^{\alpha t} \int_{t}^{\infty} s^{N-1} h_{2}(s) d s \\
< & \infty .
\end{aligned}
$$

We will now show that if $z_{t}$ is a solution of (2.9) satisfying (2.10), then there exist $\phi^{S} \in S$ such that $z_{t}$ satisfies (2.11), and, conversely, if $z_{t}$ satisfies (2.11) and (2.10) and $z_{t}$ is continuous on $t$, then $z_{t}$ satisfies (2.9).

In fact, suppose $z_{t}$ satisfies (2.9) and (2.10) with $z_{\sigma}=\phi$.

From the variation of constants formula (1.23) we have

$$
\begin{aligned}
z_{t}= & T(t-\sigma) \phi \\
& +\int_{\sigma}^{t}\left\{\left[-d s T(t-s) X_{0}^{Q}\right] G\left(s, z_{s}\right)+T(t-s) X_{0}^{Q} F\left(s, H\left(s, z_{s}\right)\right) d s\right\} \\
& +\int_{\sigma}^{t}\left\{\left[-d s T(t-s) X_{0}^{P}\right] G\left(s, z_{s}\right)+T(t-s) X_{0}^{P} F\left(s, H\left(s, z_{s}\right)\right) d s\right\} .
\end{aligned}
$$


Hence,

$$
\begin{aligned}
z_{t}= & T(t-\sigma) \bar{\phi} \\
& +\int_{\sigma}^{t}\left\{\left[-d s T(t-s) X_{0}^{Q}\right] G\left(s, z_{s}\right)+T(t-s) X_{0}^{Q} F\left(s, H\left(s, z_{s}\right)\right) d s\right\} \\
& +\int_{\sigma}^{t}\left\{\left[-d s T(t) X^{P_{1}} T(-s) X_{0}^{P}\right] G\left(s, z_{s}\right)\right. \\
& \left.\quad+T(t) X^{P_{1}} T(-s) X_{0}^{P} F\left(s, H\left(s, z_{s}\right)\right) d s\right\} \\
& -\int_{t}^{\infty}\left\{\left[-d s T(t) X^{P_{2}} T(-s) X_{0}^{P}\right] G\left(s, z_{s}\right)\right. \\
& \left.\quad+T(t) X^{P_{2}} T(-s) X_{0}^{P} F\left(s, H\left(s, z_{s}\right)\right) d s\right\} \\
\stackrel{\text { def }}{=} & T(t-\sigma) \bar{\phi}+G_{1}(t),
\end{aligned}
$$

where

$$
\begin{aligned}
\bar{\phi}=\phi+\int_{\sigma}^{\infty}\left\{\left[-d s T(\sigma) X^{P_{2}}\right.\right. & \left.T(-s) X_{0}^{P}\right] G\left(s, z_{s}\right) \\
& \left.+T(\sigma) X^{P_{2}} T(-s) X_{0}^{P} F\left(s, H\left(s, z_{s}\right)\right) d s\right\} .
\end{aligned}
$$

In order to show that $\bar{\phi} \in S$, we show that if $z_{t}$ satisfies (2.10) then $G_{1}(t)$ also satisfies

$$
\left\|G_{1}(t)\right\| / t^{l} e^{\alpha t} \rightarrow 0 \quad \text { as } t \rightarrow \infty .
$$

From Theorem 1.7 and the convergence of

$$
\left\|\int_{\sigma}^{t}\left\{\left[-d s T(t-s) X_{0}^{Q}\right] G\left(s, z_{s}\right)\right\}\right\|
$$

we have

$$
\begin{aligned}
\| \int_{\sigma}^{t}\{[ & \left.\left.-d s T(t-s) X_{0}^{Q}\right] G\left(s, z_{s}\right)+T(t-s) X_{0}^{Q} F\left(s, H\left(s, z_{s}\right)\right) d s\right\} \| \\
\leq & \int_{\sigma}^{t} e^{(\alpha-\varepsilon)(t-s)} e^{-(\alpha-\varepsilon)(t-s)}\left\|-d s T(t-s) X_{0}^{Q}\right\|\left|G\left(s, z_{s}\right)\right| \\
& \quad+\int_{\sigma}^{t}\left\|T(t-s) X_{0}^{Q}\right\|\left|F\left(s, H\left(s, z_{s}\right)\right)\right| d s \\
\leq & K e^{\alpha t} e^{-\alpha \xi} e^{-\varepsilon(t-\xi)} h_{1}(\xi)\left\|z_{\xi}\right\| \int_{0}^{t-\sigma} e^{-(\alpha-\varepsilon) u}\left\|d u T(u) X_{0}^{Q}\right\| \\
& +\bar{K} e^{\alpha t} e^{-\varepsilon t} \int_{\sigma}^{t} e^{\varepsilon s} e^{-\alpha s} h_{2}(s)\left[\left\|y_{t}\right\|+\left(1+h_{1}(t)\right)\left\|z_{t}\right\|\right] d s
\end{aligned}
$$




$$
\begin{aligned}
\leq & K t^{l} e^{\alpha t} e^{-\varepsilon(t-\xi)} h_{1}(\xi) \xi^{-l} e^{-\alpha \xi}\left\|z_{\xi}\right\| \int_{0}^{t-\sigma} e^{-(\alpha-\varepsilon) u}\left\|d u T(u) X_{0}^{Q}\right\| \\
& +\bar{K} t^{l} e^{\alpha t} e^{-\varepsilon t} \int_{\sigma}^{t} e^{\varepsilon s} s^{-l} e^{-\alpha s} h_{2}(s)\left[\left\|y_{s}\right\|+\left(1+h_{1}(s)\right)\left\|z_{s}\right\|\right] d s \\
= & K_{1} t^{l} e^{\alpha t} e^{-\varepsilon(t-\xi)} h_{1}(\xi) \int_{0}^{t-\sigma} e^{-(\alpha-\varepsilon) u}\left\|d u T(u) X_{0}^{Q}\right\| \\
& +K_{2} t^{l} e^{\alpha t} e^{-\varepsilon t} \int_{\sigma}^{t} e^{\varepsilon s} h_{2}(s) d s .
\end{aligned}
$$

From Lemma 2.2 we have

$$
\begin{aligned}
\| \int_{\sigma}^{t}\{[-d s & \left.T(t) X^{P_{1}} T(-s) X_{0}^{P}\right] G\left(s, z_{s}\right) \\
& \left.+T(t) X^{P_{1}} T(-s) X_{0}^{P} F\left(s, H\left(s, z_{s}\right)\right) d s\right\} \| \\
\leq & M t^{l-1} e^{\alpha t} \int_{\sigma}^{t} s^{N-l} e^{-\alpha s}\left|G\left(s, z_{s}\right)\right| d s \\
& +M \int_{\sigma}^{t} t^{l-1} s^{N-l} e^{\alpha(t-s)}\left|F\left(s, H\left(s, z_{s}\right)\right)\right| d s \\
\leq & \bar{M} t^{l} e^{\alpha t} \frac{1}{t} \int_{\sigma}^{t} s^{N} h_{1}(s) d s+\bar{M} t^{l} e^{\alpha t} \frac{1}{t} \int_{\sigma}^{t} s^{N} h_{2}(s) d s .
\end{aligned}
$$

In the same way we have

$$
\begin{aligned}
& \| \int_{t}^{\infty}\left\{\left[-d s T(t) X^{P_{2}} T(-s) X_{0}^{P}\right] G\left(s, z_{s}\right)\right. \\
&\left.\quad+T(t) X^{P_{2}} T(-s) X_{0}^{P} F\left(s, H\left(s, z_{s}\right)\right) d s\right\} \| \\
& \leq \int_{t}^{\infty}\left\|-d s T(t) X^{P_{2}} T(-s) X_{0}^{P}\right\|\left|G\left(s, z_{s}\right)\right| d s \\
& \quad+\int_{t}^{\infty}\left\|T(t) X^{P_{2}} T(-s) X_{0}^{P}\right\|\left|F\left(s, H\left(s, z_{s}\right)\right)\right| d s \\
& \leq M t^{l} e^{\alpha t} \int_{t}^{\infty} s^{N-l-1} e^{-\alpha s}\left|G\left(s, z_{s}\right)\right| d s \\
&+M t^{l} e^{\alpha t} \int_{t}^{\infty} s^{N-l-1} e^{-\alpha s}\left|F\left(s, H\left(s, z_{s}\right)\right)\right| d s \\
& \leq M t^{l} e^{\alpha t} \int_{t}^{\infty} s^{N-1} s^{-l} e^{-\alpha s} h_{1}(s)\left\|z_{s}\right\| d s \\
&+M t^{l} e^{\alpha t} \int_{t}^{\infty} s^{-l} e^{-\alpha s} s^{N-1} h_{2}(s)\left[\left\|y_{s}\right\|+\left(1+h_{1}(s)\right)\left\|z_{s}\right\|\right] d s \\
&= M t^{l} e^{\alpha t} \int_{t}^{\infty} s^{N-1} h_{1}(s) d s+M t^{l} e^{\alpha t} \int_{t}^{\infty} s^{N-1} h_{2}(s) d s .
\end{aligned}
$$


Then since, by hypothesis, $\lim _{t \rightarrow \infty} h_{1}(t)=0$, we have, for $t_{0}>\sigma$ large enough, $\left|h_{1}(t)\right|<M_{1}, t \geq t_{0}$. Then we can write

$$
\begin{array}{r}
\left\|G_{1}(t)\right\| \leq K_{1} t^{\prime}\left[e^{\alpha t} e^{-\varepsilon(t-\xi)} h_{1}(\xi) \int_{0}^{t-\sigma} e^{-(\alpha-\varepsilon) u}\left\|d u T(u) X_{0}^{Q}\right\|\right. \\
\quad+e^{-\varepsilon t} \int_{\sigma}^{t} e^{\varepsilon s} h_{2}(s) d s+\frac{1}{t} \int_{\sigma}^{t} s^{N} h_{1}(s) d s \\
+\frac{1}{t} \int_{\sigma}^{t} s^{N} h_{2}(s) d s+\int_{t}^{\infty} s^{N-1} h_{1}(s) d s \\
\left.+\int_{t}^{\infty} s^{N-1} h_{2}(s) d s\right] .
\end{array}
$$

We show now that $\left\|G_{1}(t)\right\| / t^{l} e^{\alpha t} \rightarrow 0$ as $t \rightarrow \infty$.

Since the first integral is bounded, $\sigma \leq \xi \leq t$ and $h_{1}(t) \rightarrow 0$, the first term of (2.12) goes to zero if $\xi \rightarrow \infty$ when $t \rightarrow \infty$ or if $\xi$ goes to a finite limit when $t \rightarrow \infty$.

From Strauss and York [11, Lemma 3.6, p. 19] it follows that

$$
e^{-\varepsilon t} \int_{\sigma}^{t} e^{\varepsilon s} h_{2}(s) d s \rightarrow 0 \quad \text { as } t \rightarrow \infty .
$$

From (2.7) it follows that

$$
\int_{t}^{\infty} s^{N-1} h_{2}(s) d s \rightarrow 0 \quad \text { as } t \rightarrow \infty
$$

and

$$
\int_{t}^{\infty} s^{N-1} h_{1}(s) d s \rightarrow 0 \quad \text { as } t \rightarrow \infty .
$$

We show finally that the terms

$$
\frac{1}{t} \int_{\sigma}^{t} s^{N} h_{i}(s) d s, \quad i=1,2,
$$

go to zero as $t \rightarrow \infty$.

By integration by parts we have

$$
\begin{aligned}
\frac{1}{t} \int_{\sigma}^{t} s^{N} h_{i}(s) d s= & \frac{1}{t}\left[\left.s \int_{\infty}^{s} \tau^{N-1} h_{i}(\tau) d \tau\right|_{\sigma} ^{t}-\int_{\sigma}^{t} d s \int_{\infty}^{s} \tau^{N-1} h_{l}(\tau) d \tau\right] \\
= & \int_{\infty}^{t} \tau^{N-1} h_{l}(\tau) d \tau-\frac{\sigma}{t} \int_{\infty}^{\sigma} \tau^{N-1} h_{l}(\tau) d \tau \\
& +\frac{1}{t} \int_{\sigma}^{t} d s \int_{s}^{\infty} \tau^{N-1} h_{l}(\tau) d \tau .
\end{aligned}
$$


From hypotheses (2.6) and (2.7) on $h_{1}$ and $h_{2}$, it follows that the first two terms of the right side of the above equality goes to zero as $t \rightarrow \infty$. Using L'Hôpital's rule we prove that the last term also goes to zero.

Thus we have proved $\left\|G_{1}(t) / t^{l} e^{\alpha t}\right\| \rightarrow 0$ as $t \rightarrow \infty$.

Conversely, suppose $z_{t}$ is a continuous solution of (2.11). Therefore $z_{t}$ satisfies

$$
\begin{aligned}
z_{t}= & T(t-\sigma) \phi \\
& +\int_{\sigma}^{t}\left\{\left[-d s T(t-s) X_{0}\right] G\left(s, z_{s}\right)+T(t-s) X_{0} F\left(s, H\left(s, z_{s}\right)\right) d s\right\},
\end{aligned}
$$

where

$$
\begin{aligned}
\phi=\phi^{S}-\int_{\sigma}^{\infty}\{[-d s & \left.T(\sigma) X^{P_{2}} T(-s) X_{0}^{P}\right] G\left(s, z_{s}\right) \\
& \left.+T(\sigma) X^{P_{2}} T(-s) X_{0}^{P} F\left(s, H\left(s, z_{s}\right)\right) d s\right\}
\end{aligned}
$$

Therefore $z(t)$ is a solution of (2.9) and satisfies $z_{\sigma}=\phi$.

Our next goal is to show that (2.11) has a continuous solution which satisfies (2.10) for $\phi^{S}$ arbitrarily fixed in $S$ and $\sigma$ large enough.

Consider the space $E$ of functions $g$ on $C([\sigma, \infty), C)$ such that $\lim _{t \rightarrow \infty}\|g(t)\| / t^{l} e^{\alpha t}=0$.

In $E$ consider the norm

$$
\|g\|_{E}=\sup _{t \geq \sigma}\|g(t)\| / t^{\prime} e^{\alpha t}
$$

One can show that $E$ with the given norm is a Banach space.

Suppose we have proved that the equation

$$
\begin{aligned}
g(t)= & T(t-\sigma) \phi^{S} \\
& +\int_{\sigma}^{t}\left\{\left[-d s T(t-s) X_{0}^{Q}\right] G(s, g(s))\right. \\
& \left.\quad+T(t-s) X_{0}^{Q} F(s, H(s, g(s))) d s\right\} \\
& +\int_{\sigma}^{t}\left\{\left[-d s T(t) X^{P_{1}} T(-s) X_{0}^{P}\right] G(s, g(s))\right. \\
& \left.\quad+T(t) X^{P_{1}} T(-s) X_{0}^{P} F(s, H(s, g(s))) d s\right\} \\
& -\int_{t}^{\infty}\left\{\left[-d s T(t) X^{P_{2}} T(-s) X_{0}^{P}\right] G(s, g(s))\right. \\
& \left.\quad+T(t) X^{P_{2}} T(-s) X_{0}^{P} F(s, H(s, g(s))) d s\right\}
\end{aligned}
$$

has a solution $g$ in $E$. 
We show that from $g$ we can find a solution $z_{t}$ of (2.11). In fact, we define

$$
z_{\sigma}=g(\sigma) \text { and } z(t)=g(t)(0), \text { for } t>\sigma .
$$

We say that $z_{t}=g(t), t \geq \sigma$.

In fact, using the same calculations given by (2.13) and putting $\phi=\phi^{S}$, one proves that

$$
\begin{array}{r}
g(t)=T(t-\sigma) \phi+\int_{\sigma}^{t}\left\{\left[-d s T(t-s) X_{0}\right] G(s, g(s))\right. \\
\left.\quad+T(t-s) X_{0} F(s, H(s, g(s))) d s\right\} \\
-\int_{\sigma}^{t}\left\{\left[-d s T(\sigma) X^{P_{2}} T(-s) X_{0}^{P}\right] G(s, g(s))\right. \\
\left.+T(\sigma) X^{P_{2}} T(-s) X_{0}^{P} F(s, H(s, g(s))) d s\right\} .
\end{array}
$$

Now the proof that $z_{t}=g(t), t \geq \sigma$, is without difficulties if we observe that for $t+\theta \geq \sigma, T(t+\theta-s) X_{0}(0)=T(t-s) X_{0}(\theta)=0$ if $t+\theta<s$ and $=I$ if $t+\theta=s$. For $t \geq \sigma$ and $s \geq \sigma+\theta$,

$$
T(t+\theta-s) X_{0}(0)=0
$$

and for $t \geq \sigma$ and $s \geq \sigma>t+\theta, T(t+\theta-s) X_{0}(0)=0$, then $z_{t}=g(t)$.

For fixed $\phi^{S}$ in $S$ and $\sigma>0$ fixed, we consider the operator $U$ defined on $E$ by if $g \in E$, therefore

$$
\begin{aligned}
(U g)(t)= & T(t-\sigma) \phi^{s} \\
& +\int_{\sigma}^{t}\left\{\left[-d s T(t-s) X_{0}^{Q}\right] G(s, g(s))\right. \\
& \left.\quad+T(t-s) X_{0}^{Q} F(s, H(s, g(s))) d s\right\} \\
& +\int_{\sigma}^{t}\left\{\left[-d s T(t) X^{P_{1}} T(-s) X_{0}^{P}\right] G(s, g(s))\right. \\
& \left.\quad+T(t) X^{P_{1}} T(-s) X_{0}^{P} F(s, H(s, g(s))) d s\right\} \\
& -\int_{t}^{\infty}\left\{\left[-d s T(t) X^{P_{2}} T(-s) X_{0}^{P}\right] G(s, g(s))\right. \\
& \left.\quad+T(t) X^{P_{2}} T(-s) X_{0}^{P} F(s, H(s, g(s))) d s\right\} .
\end{aligned}
$$

Let us now prove that $U$ has a fixed point on $E$.

First we observe that if $g$ and $w$ are elements of $E$, then, since $\phi^{S} \in S$, using (2.12) we have that there exists a constant $K_{1}>0$ such that 


$$
\begin{aligned}
\|(U g)(t)\| \leq K_{1} t^{l} e^{\alpha t}[T( & -\sigma) \phi^{S} / t^{l} e^{\alpha t} \\
& +e^{-\varepsilon(t-\xi)} h_{1}(\xi) \int_{0}^{t-\sigma} e^{-(\alpha-\varepsilon) u}\left\|d u T(u) X_{0}^{Q}\right\| \\
& +e^{-\varepsilon t} \int_{\sigma}^{t} e^{\varepsilon s} h_{2}(s) d s+\frac{1}{t} \int_{\sigma}^{t} s^{N} h_{1}(s) d s \\
& +\frac{1}{t} \int_{\sigma}^{t} s^{N} h_{2}(s) d s+\int_{t}^{\infty} s^{N-1} h_{1}(s) d s \\
& \left.+\int_{t}^{\infty} s^{N-1} h_{2}(s) d s\right] .
\end{aligned}
$$

There also exists a constant $K_{2}>0$ such that $\|(U g)(t)-(U w)(t)\|$

$$
\begin{aligned}
& \leq \| \int_{\sigma}^{t}\left\{\left[-d s T(t-s) X_{0}^{Q}\right][G(s, g(s))-G(s, w(s))]\right. \\
& \left.\quad+T(t-s) X_{0}^{Q}[F(s, H(s, g(s)))-F(s, H(s, w(s)))] d s\right\} \| \\
& +\| \int_{\sigma}^{t}\left\{\left[-d s T(t) X^{P_{1}} T(-s) X_{0}^{P}\right][G(s, g(s))-G(s, w(s))]\right. \\
& \left.\quad+T(t) X^{P_{1}} T(-s) X_{0}^{P}[F(s, H(s, g(s)))-F(s, H(s, w(s)))] d s\right\} \| \\
& +\| \int_{t}^{\infty}\left\{\left[-d s T(t) X^{P_{2}} T(-s) X_{0}^{P}\right][G(s, g(s))-G(s, w(s))]\right. \\
& \left.\quad+T(t) X^{P_{2}} T(-s) X_{0}^{P}[F(s, H(s, g(s)))-F(s, H(s, w(s)))] d s\right\} \| .
\end{aligned}
$$

By the calculations we made before we can write

$$
\begin{aligned}
& \|(U g)(t)-(U w)(t)\| \\
& \leq \bar{K}_{2} t^{l} e^{\alpha t}\|g(\xi)-w(\xi)\| \xi^{-l} e^{-\alpha \varepsilon} e^{-\varepsilon(t-\xi)} h_{1}(\xi) \\
& \quad \cdot \int_{0}^{t-\sigma} e^{-(\alpha-\varepsilon) u}\left\|d u T(u) X_{0}^{Q}\right\| \\
& \quad+\int_{\sigma}^{t} M_{3} e^{(\alpha-\gamma)(t-s)}|F(s, H(s, g(s)))-F(s, H(s, w(s)))| d s \\
& \quad+\int_{\sigma}^{t}\left\|T(t) X^{P_{1}} T(-s) X_{0}^{P}\right\||G(s, g(s))-G(s, w(s))| d s \\
& \quad+\int_{\sigma}^{t}\left\|T(t) X^{P_{1}} T(-s) X_{0}^{P}\right\||F(s, H(s, g(s)))-F(s, H(s, w(s)))| d s \\
& \quad+\int_{t}^{\infty}\left\|T(t) X^{P_{2}} T(-s) X_{0}^{P}\right\||G(s, g(s))-G(s, w(s))| d s \\
& \quad+\int_{t}^{\infty}\left\|T(t) X^{P_{2}} T(-s) X_{0}^{P}\right\||F(s, H(s, g(s)))-F(s, H(s, w(s)))| d s
\end{aligned}
$$




$$
\begin{aligned}
\leq & \bar{K}_{2} t^{l} e^{\alpha t}\|g(\xi)-w(\xi)\| \xi^{-l} e^{-\alpha \xi} e^{-\varepsilon(t-\xi)} h_{1}(\xi) \\
& \cdot \int_{0}^{t-\sigma} e^{-(\alpha-\varepsilon) u}\left\|d u T(u) X_{0}^{Q}\right\| \\
& +M_{3} t^{l} e^{\alpha t} e^{-\gamma t} \int_{\sigma}^{t} e^{\gamma s} h_{2}(s)\left[1+h_{1}(s)\right] s^{-l} e^{-\alpha s}\|g(s)-w(s)\| d s \\
& +M t^{l} e^{\alpha t} \frac{1}{t} \int_{\sigma}^{t} s^{N} h_{1}(s) s^{-l} e^{-\alpha s}\|g(s)-w(s)\| d s \\
& +M t^{l} e^{\alpha t} \frac{1}{t} \int_{\sigma}^{t} s^{N} h_{2}(s)\left[1+h_{1}(s)\right] s^{-l} e^{-\alpha s}\|g(s)-w(s)\| d s \\
& +M t^{l} e^{\alpha t} \int_{t}^{\infty} s^{N-1} h_{1}(s) s^{-l} e^{-\alpha s}\|g(s)-w(s)\| d s \\
& +M t^{l} e^{\alpha t} \int_{t}^{\infty} s^{N-1} h_{2}(s)\left[1+h_{1}(s)\right] s^{-l} e^{-\alpha s}\|g(s)-w(s)\| d s \\
\leq & \bar{K}_{2} t^{l} e^{\alpha t} e^{-\varepsilon(t-\xi)} h_{1}(\xi) \int_{0}^{t-\sigma} e^{-(\alpha-\varepsilon) u}\left\|d u T(u) X_{0}^{Q}\right\|\|g-w\|_{E} \\
& +\bar{M} t^{l} e^{\alpha t} e^{-\gamma t} \int_{\sigma}^{t} h_{2}(s) d s\|g-w\|_{E}+M t^{l} e^{\alpha t} \int_{\sigma}^{t} s^{N-1} h_{1}(s) d s\|g-w\|_{E} \\
& +\bar{M} t^{l} e^{\alpha t} \int_{\sigma}^{t} s^{N-1} h_{2}(s) d s\|g-w\|_{E}+M t^{l} e^{\alpha t} \int_{t}^{\infty} s^{N-1} h_{1}(s) d s\|g-w\|_{E} \\
& +\bar{M} t^{l} e^{\alpha t} \int_{t}^{\infty} s^{N-1} h_{2}(s) d s\|g-w\|_{E} .
\end{aligned}
$$

Thus there exists a constant $K_{2}>0$, independent of $\phi^{S}$, such that for every $t \geq \sigma$,

$$
\begin{aligned}
& \|(U g)(t)-(U w)(t)\| / t^{l} e^{\alpha t} \\
& \leq\left[\bar{K}_{2} e^{-\varepsilon(t-\xi)} h_{1}(\xi) \int_{\sigma}^{\infty} e^{-(\alpha-\varepsilon) u}\left\|d u T(u) X^{Q}\right\|\right. \\
& \quad+\bar{M}_{3} \int_{\sigma}^{\infty} h_{2}(s) d s+M \int_{\sigma}^{\infty} s^{N-1} h_{1}(s) d s \\
& \quad+\bar{M} \int_{\sigma}^{\infty} s^{N-1} h_{2}(s) d s+M \int_{\sigma}^{\infty} s^{N-1} h_{1}(s) d s \\
& \left.\quad+\bar{M} \int_{\sigma}^{\infty} s^{N-1} h_{2}(s) d s\right]\|g-w\|_{E} \\
& \leq K_{2}\left[\int_{\sigma}^{\infty} s^{N-1} h_{1}(s)+\int_{\sigma}^{\infty} s^{N-1} h_{2}(s) d s\right]\|g-w\|_{E} .
\end{aligned}
$$

From (2.17) we see that $\|(U g)(t)\| / t^{l} e^{\alpha t} \rightarrow 0$ as $t \rightarrow \infty$ and therefore $U(E) \subset E$. Furthermore, taking $\sigma$ large enough in such a way that

$$
K_{2}\left[\int_{\sigma}^{\infty} s^{N-1} h_{1}(s) d s+\int_{\sigma}^{\infty} s^{N-1} h_{2}(s) d s\right]<1,
$$


we have that $U$ will be a uniform contraction on $E$ with respect to $\phi^{S} \in S$.

Now we apply a known theorem [6], p. 7, Theorem 3.2 to conclude there exists a fixed point on $U$ which is continuous with respect to $\phi^{S}$.

For $\phi^{S}$ in $S$ and $g$ fixed in $E$, we consider

$$
\begin{aligned}
\left(U_{\phi^{s}} g\right)(t)= & T(t-\sigma) \phi^{s} \\
& +\int_{\sigma}^{t}\left\{\left[-d s T(t-s) X_{0}^{Q}\right] G(s, g(s))\right. \\
& \left.+T(t-s) X_{0}^{Q} F(s, H(s, g(s))) d s\right\} \\
& +\int_{\sigma}^{t}\left\{\left[-d s T(t) X^{P_{1}} T(-s) X_{0}^{P}\right] G(s, g(s))\right. \\
& \left.\quad+T(t) X^{P_{1}} T(-s) X_{0}^{P} F(s, H(s, g(s))) d s\right\} \\
& -\int_{t}^{\infty}\left\{\left[-d s T(t) X^{P_{2}} T(-s) X_{0}^{P}\right] G(s, g(s))\right. \\
& \left.+T(t) X^{P_{2}} T(-s) X_{0}^{P} F(s, H(s, g(s))) d s\right\} .
\end{aligned}
$$

The map $\phi^{S} \in S \rightarrow\left(U_{\phi^{s}} g\right) \in E$ is continuous in $S$. To prove it we have only to show that the map

$$
\phi^{S} \in S \rightarrow V_{\phi^{s}} \in E,
$$

where $V_{\phi} s(t)=T(t-s) \phi^{S}$, is continuous in $S$.

This follows from a simple application of the principle of uniform boundedness to the set $\left\{T(t-\sigma) / t^{l} e^{\alpha t}, t \geq \sigma\right\} \subset L(S, C)$.

Since $T(t-\sigma) \phi^{S} / t^{l} e^{\alpha t} \rightarrow 0$, when $t \rightarrow \infty$, for each $\phi^{S} \in S$, then for each $\phi^{S} \in S$ we have

$$
\sup _{t \geq \sigma}\left\|T(t-\sigma) \phi^{S} / t^{l} e^{\alpha t}\right\|<\infty, \quad \phi^{S} \in S .
$$

Then from the uniform boundedness principle

$$
\sup _{t \geq \sigma}\left\|T(t-\sigma) / t^{l} e^{\alpha t}\right\|_{L(S, C)}=\tilde{K}<\infty,
$$

and then

$$
\sup _{t \geq \sigma} \sup _{\left\|\phi^{S}\right\|=1}\left\|T(t-\sigma) \phi^{S} / t^{l} e^{\alpha t}\right\|=\tilde{K}<\infty .
$$

Then for every $t \geq \sigma$ and $\phi^{S}$ in $S$, such that $\left\|\phi^{S}\right\|=1$,

$$
\left\|T(t-\sigma) \phi^{S} / t^{l} e^{\alpha t}\right\| \leq \tilde{K},
$$

which implies

$$
\left\|T(\cdot-\sigma) \phi^{S}\right\|_{E}=\sup _{t \geq \sigma}\left\|T(t-\sigma) \phi^{S} / t^{l} e^{\alpha t}\right\| \leq \tilde{K} .
$$


Then the map $\phi^{S} \in S \rightarrow V_{\phi^{s}}=T(\cdot-\sigma) \phi^{S} \in E$ is continuous.

We now apply a well-known theorem, see Hale [5], Theorem 3.2, p. 7 with $U_{\phi^{s}}$ the uniform contraction. Then it follows that for each $\phi^{S} \in S$ there exists a unique fixed point $g\left(\phi^{S}\right) \in E$ of $U_{\phi}$. Furthermore the map $g: S \rightarrow E$ is continuous.

Then (2.11) has a continuous solution $z_{t}$ which satisfies (2.10) for $\phi^{S}$ aribtrarily fixed in $S$ and $\sigma$ large enough, that is

$$
\lim _{t \rightarrow \infty}\left\|z_{t}\right\| / t^{l} e^{\alpha t}=0 .
$$

But if $z_{t}$ is given by (2.11), $z_{t}$ satisfies (2.9) and, therefore, from (1.22) we have

$$
\bar{z}_{t}=z_{t}+X_{0} G\left(t, z_{t}\right)
$$

and then

$$
\left\|\bar{z}_{t}\right\| / t^{l} e^{\alpha t}=\left\|z_{t}+X_{0} G\left(t, z_{t}\right)\right\| / t^{l} e^{\alpha t},
$$

which implies

$$
\begin{aligned}
\left\|\bar{z}_{t}\right\| / t^{l} e^{\alpha t} & \leq\left[\left\|z_{t}\right\|+h_{1}(t)\left\|z_{t}\right\|\right] / t^{l} e^{\alpha t} \\
& =\left(1+h_{1}(t)\right)\left\|z_{t}\right\| / t^{l} e^{\alpha t} \Rightarrow \lim _{t \rightarrow \infty}\left\|z_{t}\right\| / t^{l} e^{\alpha t}=0 .
\end{aligned}
$$

But $\bar{z}_{t}=x_{t}-y_{t}$, therefore

$$
\lim _{t \rightarrow \infty}\left\|x_{t}-y_{t}\right\| / t^{l} e^{\alpha t}=0 .
$$

Now consider the map $W: S \rightarrow C$ defined by

$$
\begin{aligned}
W\left(\phi^{S}\right)=\phi^{S}-\int_{\sigma}^{\infty}\{[ & -d s \\
& \left.T(\sigma) X^{P_{2}} T(-s) X_{0}^{P}\right] G\left(s, g\left(\phi^{S}\right)(s)\right) \\
& \left.+T(\sigma) X^{P_{2}} T(-s) X_{0}^{P} F\left(s, H\left(s, g\left(\phi^{S}\right)(s)\right)\right) d s\right\} .
\end{aligned}
$$

We claim $W$ is continuous in $S$. If $\phi, \psi \in S$ we have

$$
\begin{aligned}
& \|W(\phi)-W(\psi)\| \\
& \leq\|\phi-\psi\|+\| \int_{\sigma}^{\infty}\left\{\left[-d s T(\sigma) X^{P_{2}} T(-s) X_{0}^{P}\right]\right. \\
& \cdot[G(s, g(\phi)(s))-G(s, g(\psi)(s))] \\
& +T(\sigma) X^{P_{2}} T(-s) X_{0}^{P}[F(s, H(s, g(\phi)(s))) \\
& -F(s, H(s, g(\phi)(s)))] d s\} \| .
\end{aligned}
$$


From previous calculations we have

$$
\begin{aligned}
& \|W(\phi)-W(\psi)\| \\
& \leq\|\phi-\psi\|+M \sigma^{l} e^{\alpha \sigma} \int_{\sigma}^{\infty} s^{N-1} h_{1}(s) s^{-l} e^{-\alpha s}\|g(\phi)(s)-g(\psi)(s)\| d s \\
& \quad+M \sigma^{\prime} e^{\alpha \sigma} \int_{\sigma}^{\infty} s^{N-1} h_{2}(s)\left[1+h_{1}(s)\right] s^{-l} e^{-\alpha s}\|g(\phi)(s)-g(\psi)(s)\| d s \\
& \leq\|\phi-\psi\| \\
& \quad+M \sigma^{l} e^{\alpha \sigma}\left[\int_{\sigma}^{\infty} s^{N-1} h_{1}(s) d s+K \int_{\sigma}^{\infty} s^{N-1} h_{2}(s) d s\right]\|g(\phi)-g(\psi)\|_{E} .
\end{aligned}
$$

But we already proved that the map $\phi \in S \rightarrow g(\phi) \in E$ is continuous, therefore from the above inequality it follows that $W$ is continuous.

Let $Y^{S} \stackrel{\text { def }}{=} W(S) \subset C$ and consider the map $W: S \rightarrow Y_{S}$. We shall afterwards show that the inverse $W^{-1}$ is a projection over $S$.

Let $X^{P}$ and $X^{Q}$ be the projections given by the decomposition $C=P \oplus Q$. We assert that

$$
X^{Q} W\left(\phi^{S}\right)=X^{Q} \phi^{S}
$$

In fact since the subspace $P$ is invariant under $T(t)$ for all $t \in R$, we have

$$
\begin{aligned}
\int_{\sigma}^{\infty}\left\{\left[-d s T(\sigma) X^{P_{2}} T(-s) X_{0}^{P}\right] G\left(s, g\left(\phi^{S}\right)(s)\right)\right. \\
\left.+T(\sigma) X^{P_{2}} T(-s) X_{0}^{P} F\left(s, g\left(\phi^{S}\right)(s)\right) d s\right\}
\end{aligned}
$$

is an element of $P$. Then (2.19) follows.

Using also the invariance of $P$ under $T(t)$ we have

$$
\begin{aligned}
& X^{P} W\left(\phi^{S}\right) \\
& =X^{P} \phi^{S}-\int_{\sigma}^{\infty}\left\{\left[-d s T(\sigma) X^{P_{2}} T(-s) X_{0}^{P}\right] G\left(s, g\left(\phi^{S}\right)(s)\right)\right. \\
& \left.\quad+T(\sigma) X^{P_{2}} T(-s) X_{0}^{P} F\left(s, H\left(s, g\left(\phi^{S}\right)(s)\right)\right) d s\right\} .
\end{aligned}
$$

Thus

$$
\begin{aligned}
& T(-\sigma) X^{P} W\left(\phi^{S}\right) \\
& =T(-\sigma) X^{P} \phi^{S}-\int_{\sigma}^{\infty}\left\{\left[\begin{array}{l}
\left.-d s X^{P_{2}} T(-s) X_{0}^{P}\right] G\left(s, g\left(\phi^{S}\right)(s)\right) \\
\quad \\
\left.\quad+X^{P_{2}} T(-s) X_{0}^{P} F\left(s, H\left(s, g\left(\phi^{S}\right)(s)\right)\right) d s\right\} .
\end{array}\right.\right.
\end{aligned}
$$


We remark now that $X^{P} \phi^{S} \in P_{1}$. Furthermore $P_{1}$ is invariant under $T(t)$. We also have that $P_{2}$ is not invariant in general under $T(t)$. In general it is not also true that $T(t) X^{P_{1}}=X^{P_{1}} T(t)$.

From the remarks above it follows that

$$
X^{P_{1}} T(-\sigma) X^{P} W\left(\phi^{S}\right)=X^{P_{1}} T(-\sigma) X^{P} \phi^{S}=T(-\sigma) X^{P} \phi^{S} \in P_{1} .
$$

Then

$$
T(\sigma) X^{P_{1}} T(-\sigma) X^{P} W\left(\phi^{S}\right)=X^{P} \phi^{S} .
$$

From (2.19) and (2.20) it follows that

$$
\left[T(\sigma) X^{P_{1}} T(-\sigma) X^{P}+X^{Q}\right] W\left(\phi^{S}\right)=X^{P} \phi^{S}+X^{Q} \phi^{S}=\phi^{S} .
$$

Therefore we conclude that $W^{-1}: Y_{S} \rightarrow S$ is

$$
\left.\left[T(\sigma) X^{P_{1}} T(-\sigma) X^{P}+X^{Q}\right]\right|_{Y_{S}} \text {. }
$$

We now show that this map is a projection. In fact

$$
\begin{aligned}
& \left(T(\sigma) X^{P_{1}} T(-\sigma) X^{P}\right)\left(T(\sigma) X^{P_{1}} T(-\sigma) X^{P}\right) \\
& \quad=T(\sigma) X^{P_{1}} T(-\sigma) T(\sigma) X^{P_{1}} T(-\sigma) X^{P}=T(\sigma) X^{P_{1}} T(-\sigma) X^{P} .
\end{aligned}
$$

Thus we conclude that $T(\sigma) X^{P_{1}} T(-\sigma) X^{P}+X^{Q}$ is a projection. Furthermore if $\phi \in C$, then $\left[T(\sigma) X^{P_{1}} T(-\sigma) X^{P}+X^{Q}\right] \phi$ belongs to $S$. We now show that it is a projection over $S$.

In fact if $\phi \in S$ we have $\phi=\phi^{Q}+\phi^{P_{1}}$ and then

$$
\begin{aligned}
& {\left[T(\sigma) X^{P_{1}} T(-\sigma) X^{P}+X^{Q}\right]\left(\phi^{Q}+\phi^{P_{1}}\right)} \\
& \quad=T(\sigma) X^{P_{1}} T(-\sigma) X^{P^{P_{1}}}+X^{Q} \phi^{Q}=T(\sigma) T(-\sigma) \phi^{P_{1}}+\phi^{Q}=\phi .
\end{aligned}
$$

The continuity of this projection follows from the fact that $X^{Q}, X^{P_{1}}$ and $T(t)$ are continuous. Therefore we proved that $W$ is an homeomorphism.

For ordinary differential equations we can prove the converse of Theorem 2.3, that is, for each solution $x_{t}$ of $(P)$ there is a solution $y_{t}$ of $(\mathrm{L})$ such that $\left\|x_{t}-y_{t}\right\| /\left\|x_{t}\right\| \rightarrow 0$ when $t \rightarrow \infty$. For delay equations this is no longer true as is shown by the following example given by J. Hale. Consider the equation

$$
\dot{y}=0 \text { and } \dot{x}=-2 t \exp (1-2 t(x(t-1))),
$$

which has the solution $x(t)=\exp \left(-t^{2}\right)$ and $\left[1-\exp \left(-t^{2}\right)\right] / \exp \left(-t^{2}\right) \nrightarrow 0$ as $t \rightarrow \infty$.

However it is possible to give a partial converse of Theorem 2.3, that is, if the Lyapunov numbers of the solutions of (L) are finite then the converse is true. This was proved for retarded functional differential 
equations by Rodrigues [10]. We give in the following the extension of these results to neutral equations. We will need the following lemmas:

LEMMA 2.4. Let $\rho, g \in L_{1}((0, \infty), R), \rho, g \geq 0$. Let $\gamma(t) \geq 0$ be $a$ decreasing smooth function, $\gamma(t) \rightarrow 0$ as $t \rightarrow \infty$. Let $u(t) \geq 0$ be a continuous solution of

$$
u(t) \leq K+\int_{\sigma}^{t} u(s) \rho(s) d s+\frac{1}{\gamma(t)} \int_{t}^{\infty} \gamma(s) u(s) g(s) d s, \quad t \geq \sigma,
$$

such that $\gamma(t) u(t)$ is bounded. Then

$$
u(t) \leq \frac{K}{1-\beta} \exp \left\{\frac{1}{1-\beta} \int_{t}^{\infty} g(s) d s\right\},
$$

where $\beta=\int_{\sigma}^{\infty}[\rho(s)+g(s)] d s<1$.

Proof. Let $V(t) \stackrel{\text { def }}{=} \max _{0 \leq s \leq t} u(s)$. Then $V$ is continuous, decreasing, $u(t) \leq V(t)$ and $\gamma(t) V(t)$ is bounded.

For given $t \geq \sigma$ there exists $t_{1} \in[\sigma, t]$ such that

$$
\begin{aligned}
V(t)= & u\left(t_{1}\right) \leq K+V(t)\left[\int_{\sigma}^{\infty} \rho(s) d s+\int_{\sigma}^{\infty} g(s) d s\right] \\
& +\frac{1}{\gamma(t)} \int_{t}^{\infty} \gamma(s) V(s) g(s) d s .
\end{aligned}
$$

Let $\sigma$ be sufficiently large in such a way that $\beta<1$. Then

$$
\gamma(t) V(t) \leq \frac{1}{1-\beta}\left[K \gamma(t)+\int_{t}^{\infty} \gamma(s) V(s) g(s) d s\right],
$$

and from Gronwall's inequality we have

$$
\gamma(t) V(t) \leq \frac{K}{1-\beta} \gamma(t) \exp \left\{\frac{1}{1-\beta} \int_{t}^{\infty} g(s) d s\right\} .
$$

LEMMA 2.5. Let $x(t)$ be a solution of $(\mathrm{P})$ such that

$$
\varlimsup_{t \rightarrow \infty} \frac{\log \left\|x_{t}\right\|}{t}=\mu \in R, \quad u>a_{D},
$$

where

$$
\begin{gathered}
|f(t, \Phi)| \leq h_{2}(t)\|\phi\|, \quad|G(t, \phi)| \leq h_{1}(t)\|\phi\|, \\
\int^{\infty} h_{1}(t) d t<\infty, \quad \int^{\infty} h_{2}(t) d t<\infty, \quad \lim h_{1}(t)=0 .
\end{gathered}
$$

Then there exists $\lambda \in \sigma(A)$ such that $\operatorname{Re} \lambda=\mu$. 
Proof. Assume that for every $\lambda \in \sigma(A), \operatorname{Re} \lambda \neq \mu$. Let $\Lambda=\{\lambda \in$ $\sigma(A) \mid \operatorname{Re} \lambda>\mu\}$. Then $C=P \oplus Q$ and there exists $\varepsilon>0$ such that

$$
\begin{gathered}
\left\|T(t-\sigma) \phi^{Q}\right\| \leq M_{1} e^{(\mu-\varepsilon)(t-\sigma)}\left\|\phi^{Q}\right\|, \quad t \geq \sigma, \quad \phi^{Q} \in Q, \\
\left\|T(t-\sigma) \phi^{P}\right\| \leq M_{2} e^{(\mu-\varepsilon)(t+\sigma)}\left\|\phi^{P}\right\|, \quad t \geq \sigma, \quad \phi^{P} \in P, \\
\left\|T(t-\sigma) X_{0}^{P}\right\| \leq M_{3} e^{(\mu-\varepsilon)(t-\sigma)}, \quad t \leq \sigma, \\
\left\|T(t-\sigma) X_{0}^{Q}\right\| \leq M_{4} e^{(\mu-\varepsilon)(t-\sigma)}, \quad t \geq \sigma, \\
\int^{\infty}\left\|d T(s) X_{0}^{Q}\right\| e^{-(\mu-\varepsilon) s} \leq M_{3}, \\
\int^{\infty}\left\|d T(s) X_{0}^{P}\right\| e^{-(\mu+\varepsilon) s} \leq M_{4} .
\end{gathered}
$$

Using the variation of constants formula

$$
\begin{aligned}
z_{t}=T(t-\sigma) \Phi+\int_{\sigma}^{t}\left\{\left[-d s T(t-s) X_{0}^{Q}\right] G\left(s, z_{s}\right)\right. \\
\left.\quad+T(t-s) X_{0}^{Q} F\left(s, H\left(s, z_{s}\right)\right) d s\right\} \\
-\int_{t}^{\infty}\left\{\left[-d s T(t-s) X_{0}^{P}\right] G\left(s, z_{s}\right)+T(t-s) X_{0}^{P} F\left(s, z_{s}\right) d s\right\}
\end{aligned}
$$

where

$$
\begin{aligned}
\Phi=\phi+\int_{\sigma}^{\infty}\{[ & \left.-d s T(t-s) X_{0}^{P}\right] G\left(s, z_{s}\right) \\
& \left.+T(t-s) X_{0}^{P} F\left(s, H\left(s, z_{s}\right)\right) d s\right\} .
\end{aligned}
$$

From the Mean Value Theorem we have

$$
\begin{aligned}
e^{-(\mu-\varepsilon) t}\left|\int_{\sigma}^{t}\left[-d s T(t-s) X_{0}^{Q}\right] G\left(s, x_{s}\right) d s\right| \\
\leq e^{-(\mu-\varepsilon)(t-\sigma)}\left|e^{(\mu-\varepsilon)(t-\sigma)} K_{1} h_{1}(\xi) \int_{t}^{t-\sigma} e^{-(\mu-\varepsilon) u} \| d u T(u) X_{0}^{Q \|}\right| \\
\leq K e^{-2 \varepsilon t} e^{\varepsilon \sigma+\varepsilon \xi} h_{1}(\xi)=K e^{-\varepsilon(t-\sigma)} e^{-\varepsilon(t-\xi)} h_{1}(\xi), \quad \sigma \leq \xi \leq t .
\end{aligned}
$$

If $\xi$ goes to a constant or infinity, the above expression goes to zero. Since

$$
d s T(t-s) X_{0}^{P}=B e^{B(t-s)} X_{0}^{P}
$$

and

$$
\mid F\left(t, H\left(t, z_{t}\right) \mid \leq h_{2}(t)\left[1+h_{1}(t)\right]\left\|z_{t}\right\|,\right.
$$


we can prove that

$$
\begin{array}{r}
e^{-(\mu+\varepsilon) t} \mid \int_{\sigma}\left\{\left[-d s T(t-s) X_{0}^{Q}\right] G\left(s, z_{s}\right)+T(t-s) X_{0}^{Q} F\left(s, H\left(s, z_{s}\right)\right) d s\right\} \\
+\int^{\infty}\left\{\left[-d s T(t-s) X_{0}^{P}\right] G\left(s, z_{s}\right)\right. \\
\left.+T(t-s) X_{0}^{P} F\left(s, H\left(s, z_{s}\right)\right) d s\right\} \mid
\end{array}
$$

goes to zero.

Since $\mu$ being the Lyapunov number of $x_{t}$ implies $e^{-(\mu+\varepsilon) t}\left|x_{t}\right| \rightarrow 0$ as $t \rightarrow \infty$, then $e^{-(\mu+\varepsilon) t} T(t-\sigma) \Phi$ also goes to zero when $t \rightarrow \infty$. Since we assumed $\mu$ does not belong to $\sigma(A)$ and $\mu>a_{D}$, there is $\varepsilon>0$ such that $\mu-\varepsilon$ also does not belong to $\sigma(A)$ and, since $e^{-(\mu+\varepsilon) t} T(t-\sigma) \phi \rightarrow 0, \phi$ does not belong to $P$, and from the relations above $\|T(t-\sigma) \phi\| \leq M e^{(\mu-\varepsilon)(t-\sigma)}\|\phi\| e^{-(\mu-\varepsilon)(t-\sigma)}$

$$
\begin{gathered}
\cdot\left|\int_{\sigma}\left[-d s T(t-s) X_{0}^{Q}\right] G\left(s, x_{s}\right) d s\right| \\
\leq K e^{-\varepsilon(t-\xi)} h_{2}(\xi) \leq K e^{-(\mu-\varepsilon)(t-\sigma)}\left|\int_{\sigma}^{t} T(t-s) X_{0}^{Q} F\left(s, H\left(s, z_{s}\right)\right) d s\right| \\
\leq e^{-(\mu-\varepsilon)(t-\sigma)} \int_{\sigma}^{t} M_{4} e^{(\mu-\varepsilon)(t-s)} F\left(s, H\left(s, z_{s}\right)\right) d s \\
\leq K \int_{\sigma}^{t} h_{2}(s)\left(1+h_{1}(s)\right)\left\|z_{s}\right\| e^{-(\mu-\varepsilon) s} d s . \\
\quad e^{-(\mu-\varepsilon)(t-\sigma)}\left|\int_{t}^{\infty}\left[-d s T(t-s) X_{0}^{P}\right] G\left(s, z_{s}\right)\right| \\
\leq K e^{-(\mu-\varepsilon)(t-\sigma)} \int_{t}^{\infty} B e^{B(t-s)} h_{1}(s)\left\|z_{s}\right\| \\
\leq K e^{-(\mu-\varepsilon)(t-\sigma)} \int_{t}^{\infty} e^{(\mu+\varepsilon)(t-s)} h_{1}\left\|z_{s}\right\| \\
\leq K \int_{t}^{\infty} e^{2 \varepsilon t} e^{-2 \varepsilon s} h_{1}(s)\left\|z_{s}\right\| e^{-(\mu-\varepsilon) s} d s .
\end{gathered}
$$

Then

$$
\begin{aligned}
\left|z_{s}\right| e^{-(\mu-\varepsilon) t} \leq & K+K_{1} \int_{\sigma}^{t} h_{1}(s)\left(1+h_{1}(s)\right)\left|z_{s}\right| e^{-(\mu-\varepsilon) s} d s \\
& +e^{2 \varepsilon t} K_{2} \int_{t}^{\infty} e^{-2 \varepsilon s} h_{1}(s)\left|z_{s}\right| e^{-(\mu-\varepsilon)} d s \\
= & K+K_{2} \int_{\sigma}^{t} \rho(s)\left|z_{s}\right| e^{-(\mu-\varepsilon) s} d s \\
& +K_{2} e^{2 \varepsilon t} \int_{t}^{\infty} e^{-2 \varepsilon s} g(s)\left|z_{s}\right| e^{-(\mu-\varepsilon) s} d s
\end{aligned}
$$


From Lemma 2.4, $z_{t} e^{-(\mu-\varepsilon) s}$ is bounded, a contradiction, because $\mu$ is the Lyapunov number of $z_{t}$ and then the Theorem is proved.

LEMMA 2.6. Let $\mu>a_{D}$ and let $x(t)$ be a solution of $(\mathrm{P})$ such that

$$
\varlimsup \frac{\log \left|x_{t}\right|}{t}=\mu \in R
$$

and assume condition (2.21) is satisfied. Then there exists a nonnegative integer l such that

$$
0<C_{1} \leq \frac{\left|x_{t}\right|}{t^{\prime} e^{\mu t}} \leq C_{2}<\infty
$$

Proof. Let

$$
\begin{aligned}
& \Lambda_{p}=\{\lambda \in \sigma(A) \mid \operatorname{Re} \lambda=\mu\}, \\
& \Lambda_{S}=\{\lambda \in \sigma(A) \mid \operatorname{Re} \lambda>\mu\}, \\
& \Lambda_{Q}=\{\lambda \in \sigma(A) \mid \operatorname{Re} \lambda<\mu\} .
\end{aligned}
$$

Then $C=P \oplus S \oplus Q$. As before let $P_{1}=\left\{\phi \in P \mid T(t) \phi / t e^{\mu t} \rightarrow 0\right\}$ where $l \geq 0$ is fixed. We can choose $P_{2}, \sigma>0$ in such a way that $P=P_{1} \oplus P_{2}$. See E. A. Coddington and N. Levinson, Theory of Ordinary Differential Equations, McGraw-Hill, 1955, Exercise 33, p. 106.

$$
\begin{array}{ll}
\left|T(t) X^{P_{1}} T(-s) \phi^{P}\right| \leq K t^{l-1} s^{N-l} e^{\mu(t-s)}\left|\phi^{P}\right|, & \sigma \leq s \leq t, \\
\left|T(t) X^{P_{2}} T(-s) \phi^{P}\right| \leq K t^{l} s^{N-l-1} e^{\mu(t-s)}\left|\phi^{P}\right|, & \sigma \leq t \leq s .
\end{array}
$$

Moreover there exists $\varepsilon>0$ such that

$$
\begin{aligned}
\left|T(t-s) \phi^{Q}\right| & \leq K e^{(\mu-\varepsilon)(t-s)}\left|\phi^{Q}\right|, & & \sigma \leq s \leq t, \\
\left|T(t-s) \phi^{S}\right| & \leq K e^{(\mu+\varepsilon)(t-s)}\left|\phi^{Q}\right|, & & \sigma \leq t \leq s .
\end{aligned}
$$

Let $\tilde{Q} \stackrel{\text { def }}{=} Q+P$. The following estimates hold provided $\varepsilon>0$ is small enough:

$$
\begin{aligned}
\left|T(t) \phi^{\tilde{Q}}\right| & \leq K e^{(\mu-\varepsilon / 2) t}\left|\phi^{\tilde{Q}}\right|, & & t \geq 0 \\
\left|T(t) \phi^{S}\right| & \leq K e^{(\mu+\varepsilon) t}\left|\phi^{S}\right|, & & t \leq 0
\end{aligned}
$$


Since

$$
\begin{aligned}
z_{t}= & T(t-\sigma) \phi \\
& +\int_{\sigma}^{t}\left\{\left[-d s T(t-s) X_{0}^{\tilde{Q}}\right] G\left(s, x_{s}\right)+T(t-s) X_{0}^{\tilde{Q}} F\left(s, H\left(s, z_{s}\right)\right) d s\right\} \\
& -\int_{t}^{\infty}\left\{\left[-d s T(t-s) X_{0}^{S}\right] G\left(s, z_{s}\right)+T(t-s) X_{0}^{S} F\left(s, H\left(s, z_{s}\right)\right) d s\right\} .
\end{aligned}
$$

From the above estimates it is easy to show that

$$
\lim _{t \rightarrow \infty} e^{-(\mu+\varepsilon) t} T(t-\sigma) \phi \rightarrow 0 .
$$

It then follows that $\phi^{S}=0$. We can also prove, using Lemma 2.4 and the above estimates, that $|T(t-\sigma) \phi| / t^{N-1} e^{\mu t}$ is bounded, where $N=$ largest order of the blocks of $B$, where $B$ is in the Jordan canonical form and $T(t) \phi^{S}=e^{B t}(\Phi, \Psi)$.

Let $l=\min \left\{n \geq 0:\left|x_{t}\right| / t^{N} e^{\mu t}\right.$ be bounded for $\left.t \geq \sigma\right\}$.

We can prove, as we did before, that $z_{t}$ can be written in the following form:

$$
\begin{aligned}
z_{t}= & T(t-\sigma) \Psi \\
& +\int_{\sigma}^{t}\left\{\left[-d s T(t-s) X_{0}^{Q}\right] G\left(s, z_{s}\right)+T(t-s) X_{0}^{Q} F\left(s, H\left(s, z_{s}\right)\right) d s\right\} \\
& +\int_{\sigma}^{\infty}\left\{\left[-d s T(t) X^{P_{1}} T(-s) X_{0}^{P}\right] G\left(s, z_{s}\right)\right. \\
& \left.+T(t) X^{P_{1}} T(-s) X_{0}^{P} F\left(s, H\left(s, z_{s}\right)\right) d s\right\} \\
& -\int_{\sigma}^{\infty}\left\{\left[-d s T(t) X^{P_{2}} T(-s) X_{0}^{P}\right] G\left(s, z_{s}\right)\right. \\
& \left.+T(t) X^{P_{2}} T(-s) X_{0}^{P} F\left(s, H\left(s, z_{s}\right)\right) d s\right\},
\end{aligned}
$$

where

$$
\begin{aligned}
\psi=\phi^{Q}+\int_{\sigma}^{\infty}\left\{\left[-d s T(\sigma) X^{P_{2}}\right.\right. & \left.T(-s) X_{0}^{P}\right] G\left(s, z_{s}\right) \\
& \left.+T(\sigma) X^{P_{2}} T(-s) F\left(s, z_{s}\right) d s\right\} .
\end{aligned}
$$

It only remains to prove that the above integrals are convergent, and it also follows that $t^{-l} e^{-\mu t} T(t-\sigma) \psi$ is bounded. Now this is not difficult, using Lemma 2.4 and the known estimates that $t^{-t} e^{-\mu t} T(t-\sigma) \psi \nrightarrow 0$ as $t \rightarrow \infty$ and there exists $C_{1}$ such that $|T(t-\sigma) \psi| / t^{l} e^{\mu t} \geq C_{1}>0$ for every $t$ large enough.

Our final conclusion is that there exist constants $C_{1}, C_{2}$ such that $0<C_{1} \leq x_{t} / t^{l} e^{\mu t} \leq C_{2}<\infty$. We then have the following theorem. 
THEOREM 2.7. Assume $f$ and $G$ are continuous. Let $x(t) \neq 0$ for all sufficiently large $t$ be a solution of $(\mathrm{P})$. Let

$$
\lim _{t \rightarrow \infty} \frac{\log \left|x_{t}\right|}{t}=\mu \in R \text {. }
$$

Let $N=\max \left\{m /(A-\lambda I)^{m+1}=(A-\lambda I)^{m}, \operatorname{Re} \lambda=\mu\right\}$. Let us assume that

$$
\begin{gathered}
|f(t, \phi)| \leq h_{2}(t)\|\phi\|, \quad|G(t, \phi)| \leq h_{1}(t)\|\phi\|, \\
\int^{\infty} t^{N-1} h_{1}(t) d t<\infty, \quad \int^{\infty} t^{N-1} h_{2}(t) d t<\infty, \quad \lim _{t \rightarrow \infty} h_{1}(t)=0 .
\end{gathered}
$$

Then there exists a family of solutions $y_{t}$ of $(\mathrm{L})$ such that

$$
\lim _{t \rightarrow \infty} \frac{\left\|x_{t}-y_{t}\right\|}{\left\|x_{t}\right\|}=0
$$

The proof follows easily from the estimates given before, Lemma 2.4 and the change of variables (1.22).

Applications. Consider the equation

(P) $\frac{d}{d t}\left[x(t)-\alpha_{0} x(t-r)\right]=\beta x(t)-\alpha_{0} \gamma x(t-r)+b(t) x(t-r)$, where

$$
\begin{gathered}
D \phi=\phi(0)-\alpha_{0} \phi(-r), \quad L \phi=\beta \phi(0)-\alpha_{0} \gamma \phi(-r), \quad G(t, \phi) \equiv 0 \\
|F(t, \phi)|=|b(t) \phi(-r)| \leq|b(t)|\|\phi\| \quad \text { and } \quad \int^{\infty} b(t) d t<\infty
\end{gathered}
$$

The associated linear equation

$$
\frac{d}{d t}\left[x(t)-\alpha_{0} x(t-r)\right]=\beta x(t)-\alpha_{0} \gamma x(t-r)
$$

was given by R. Brayton, Bifurcation of periodic solutions in a nonlinear difference-differential equation, Quart. Appl. Math. 24 (1966). In the study of transmission lines Brayton showed that for $\gamma>\beta>0$ there exists an infinite set of real couples $\left(\alpha_{0}, w_{0}\right), w_{0}>0, \alpha_{0}^{2}<1$, such that $\pm i w_{0}$ are simple roots of the characteristic equation

$$
\lambda-\alpha_{0} \lambda e^{-r}+\beta+\alpha_{0} \gamma e^{-\lambda r}=0,
$$

and $w_{0}, \alpha_{0}$ satisfy the formulas

$$
\sin w_{0} r=\frac{w_{0}}{\alpha_{0}} \cdot \frac{\gamma+\beta}{w_{0}^{2}+\gamma^{2}}, \quad \cos w_{0} r=\frac{1}{\alpha_{0}} \cdot \frac{w_{0}^{2}-\gamma \beta}{w_{0}^{2}+\gamma^{2}} .
$$


We assume, in systems (L) and (P), $\alpha_{0}=\frac{1}{2}, \beta=1, \alpha_{0} \gamma=3, r=1$. If $\alpha_{0}=\frac{1}{2}<1$, by Melvin's theorem the operator $D$ is stable. See [5].

We now determine the decomposition of $C$ by $\Lambda=\left\{+i w_{0},-i w_{0}\right\}$.

If $\Phi=\left(\phi_{1}, \phi_{2}\right), \phi_{1}(\theta)=\sin w_{0} \theta, \phi_{2}(\theta)=\cos w_{0} \theta,-r \leq \theta \leq 0$, then $\Phi$ is a base for the generalized eigenspace of (L) associated with $\Lambda$, since we are assuming these eigenvalues are simple.

Furthermore, $A \Phi=\Phi B$ implies

$$
B=\left(b_{\imath \jmath}\right), \quad b_{11}=b_{22}=0, \quad b_{12}=-b_{21}=-w_{0} .
$$

The bilinear form associated to $(\mathrm{P})$ is

$$
\begin{aligned}
(\psi, \phi)= & \psi(0) \phi(0)-\alpha_{0} \psi(0) \phi(-r) \\
& -\alpha_{0} \int_{-r}^{0} \dot{\psi}(\theta+r) \phi(\theta) d \theta-\alpha_{0} \gamma \int_{-r}^{0} \psi(\theta+r) \phi(\theta) d \theta
\end{aligned}
$$

the adjoint equation is

$$
\frac{d}{d t}\left[y(t)-\alpha_{0} y(t+r)\right]=\beta y(t)+\alpha_{0} \gamma y(t+r),
$$

and

$$
\Psi^{*}=\operatorname{col}\left(\psi_{1}^{*}, \psi_{2}^{*}\right), \quad \psi_{1}^{*}(\theta)=\sin w_{0} \theta, \quad \psi_{2}^{*}(\theta)=\cos w_{0} \theta,
$$

$0 \leq \theta \leq r$, is a base for generalized real eigenspace of the adjoint equation associated with $\Lambda$.

One can show that

$$
\begin{aligned}
& \left(\psi_{1}^{*}, \phi_{1}\right)=\left(\psi_{2}^{*}, \phi_{2}\right)=\frac{1}{2\left(w_{0}^{2}+\gamma^{2}\right)}\left[\gamma(\gamma+\beta)+r \beta\left(\gamma^{2}+w_{0}^{2}\right)\right], \\
& \left(\psi_{2}^{*}, \phi_{1}\right)=-\left(\psi_{1}^{*}, \phi_{2}\right)=\frac{w_{0}}{2\left(w_{0}^{2}+\gamma^{2}\right)}\left[\gamma+\beta+r\left(\gamma^{2}+w_{0}^{2}\right)\right] .
\end{aligned}
$$

If we define

$$
\Psi=\left(\Psi^{*}, \Phi\right)^{-1} \Psi^{*}
$$

then $(\Psi, \Phi)=I$ and we can make the decomposition of $C$ by $\Lambda$.

From the above assumptions we have that the set $\{\lambda \in \sigma(A)$ : $\operatorname{det} \Delta(\lambda)=0\}$ satisfies $\operatorname{Re} \lambda \leq 0$ and the $\lambda \in \sigma(A)$ such that $\operatorname{Re} \lambda=0$ are

$$
\lambda_{1}=+i w_{0} \text { and } \lambda_{2}=-i w_{0} .
$$

By Theorem 1.9, since $D$ is stable there exists $\delta>0$ such that $\operatorname{Re} \lambda \leq-\delta$, with $\lambda$ the solution of

$$
\operatorname{det}\left[I-\int_{-r}^{0} e^{\lambda \theta} d \mu(\theta)\right]=0 .
$$


Then we have that there are only two eigenvalues with real part equal to zero and there exists $\gamma>0$ such that the set $\{z: 0<|\operatorname{Re} z|<\gamma\}$ does not contain any eigenvalue.

If we put $x_{t}=x_{t}(\sigma, \phi, h)=x_{t}^{P}+x_{t}^{0}$ and if $x_{t}^{P}(\sigma, \phi, h)=\Phi y(t)$, then $y(t)$ satisfies the ordinary differential equation

$$
\dot{y}(t)=B_{\Lambda} y(t)+\Psi(0) h(t), \quad t \geq \sigma,
$$

where $B_{\Lambda}=B$ and

$$
\Psi(0)=\operatorname{col}\left(\frac{D}{E^{2}+D^{2}}, \frac{1}{E^{2}+D^{2}}\right)
$$

with

$$
\begin{aligned}
& E=\frac{1}{2\left(w_{0}^{2}+\gamma^{2}\right)}\left[\gamma(\gamma+\beta)+r \beta\left(\gamma^{2}+w_{0}^{2}\right)\right], \\
& D=\frac{w_{0}}{2\left(w_{0}^{2}+\gamma^{2}\right)}\left[\gamma+\beta+r\left(\gamma^{2}+w_{0}^{2}\right)\right] .
\end{aligned}
$$

If

$$
B=\left[\begin{array}{cc}
0 & w_{0} \\
-w_{0} & 0
\end{array}\right]
$$

we have

$$
e^{B t}=\left[\begin{array}{cc}
0 & e^{i w_{0} t} \\
-e^{l w_{0} t} & 0
\end{array}\right]
$$

and then $\left[\begin{array}{l}1 \\ 1\end{array}\right]$ and $\left[{ }^{-1} 1\right]$ are the respective eigenvectors associated to $+i w_{0}$ and $-i w_{0}$.

Furthermore,

$$
y_{1}=\left[\begin{array}{c}
e^{i w_{0} t} \\
i e^{i w_{0} t}
\end{array}\right] \text { and } y_{2}=\left[\begin{array}{c}
-e^{-i w_{0} t} \\
i e^{-l w_{0} t}
\end{array}\right]
$$

are solutions of

$$
\dot{y}(t)=B_{\Lambda} y(t) .
$$

Since the conditions of Theorem 2.1 are satisfied we conclude that

$$
\frac{x(t)-C_{1} e^{l w_{0} t}}{C_{1} e^{l w_{0} t}} \rightarrow 0 \quad \text { with } t \rightarrow \infty,
$$

and this implies

$$
x(t)-C_{1} e^{l w_{0} t}=C_{1} e^{l w_{0} t} \cdot o(1)=o(1) .
$$




\section{REFERENCES}

[1] R. Bellman and K. Dooke, Asymptotic behavior of solutions of differential-difference equations, Memoirs of the Amer. Math. Soc., 35 (1959).

[2] W. Brumley, On the asymptotic behavior of solutions of differential-difference equations of the neutral type, J. Differential Equations, 7 (1970), 175-188.

[3] L. Cooke, Asymptotic equivalence of an ordinary and a functional differential equations, J. Math. Anal. Appl., 51 (1975), 187-207.

[4] P. S. Gromova and A. M. Sverkin, On trigonometric series whose sums are continuous unbounded functional on the real line, Differentialniye Uravnieniya, 4 (1968), 17741784.

[5] J. K. Hale, Theory of Functional Differential Equations, Springer-Verlag, N.Y., Heidelberg, Berlin, (1977).

[6] _ Ordinary Differential Equations, Wiley-Interscience, (1969).

[7] Linear asymptotically autonomous functional differential equations, Rend. Circ. Mat. Palermo, (2) 15 (1966), 331-351.

[8] D. Henry, Linear autonomous neutral functional differential equations, J. Differential Equations, 15 (1974), 106-128.

[9] A. F. Izé and N. A. Molfetta, Asymptotically autonomous neutral functional differential equations with time-dependent lag, J. Math. Anal. Appl., 51, no. 2, (1975).

[10] H. M. Rodrigues, On growth and decay of solutions of perturbed retarded linear equations, Tôhoku Math. J., 32, no. 4, (1980).

[11] A. Strauss and J. A. Yorke, Perturbation on theorem for ordinary differential equations, J. Differential Equations, 3 (1967), 18, p. 19.

Received April 2, 1981 and in revised form June 14, 1982. This research was supported by CNPq, FAPESP and FINEP. This paper has been printed under financial support of F.A.P.E.S.P.

\section{ICMSC-USP}

DEPARTAMENTO DE MATEMÁtica

13.560-SÃo CARLOS-SP

BRAZIL 



\section{PACIFIC JOURNAL OF MATHEMATICS}

EDITORS

Donald BabBITT (Managing Editor)

University of California

Los Angeles, CA 90024

Hugo Rossi

University of Utah

Salt Lake City, UT 84112

C. C. Moore and Arthur Ogus

University of California

Berkeley, CA 94720
J. DugunduI

Department of Mathematics

University of Southern California

Los Angeles, CA 90089-1113

R. FINN and H. SAMELSON

Stanford University

Stanford, CA 94305

\section{ASSOCIATE EDITORS}
R. ARENS
E. F. BECKENBACH
B. H. NeumanN
F. WolF
K. YosHIDA (1906-1982)

\section{SUPPORTING INSTITUTIONS}

UNIVERSITY OF ARIZONA

UNIVERSITY OF BRITISH COLUMBIA

CALIFORNIA INSTITUTE OF TECHNOLOGY

UNIVERSITY OF CALIFORNIA

MONTANA STATE UNIVERSITY

UNIVERSITY OF NEVADA. RENO

NEW MEXICO STATE UNIVERSITY

OREGON STATE UNIVERSITY
UNIVERSITY OF OREGON

UNIVERSITY OF SOUTHERN CALIFORNIA

STANFORD UNIVERSITY

UNIVERSITY OF HAWAII

UNIVERSITY OF TOKYO

UNIVERSITY OF UTAH

WASHINGTON STATE UNIVERSITY

UNIVERSITY OF WASHINGTON 


\section{Pacific Journal of Mathematics}

\section{Vol. 111, No. $1 \quad$ November, 1984}

Harald Brandenburg and Adam Stefan Mysior, For every Hausdorff

space $Y$ there exists a nontrivial Moore space on which all continuous

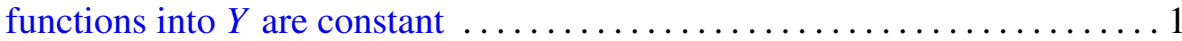

Henry Dappa, A Marcinkiewicz criterion for $L^{p}$-multipliers $\ldots \ldots \ldots \ldots \ldots 9$

P. H. Doyle, III and John Gilbert Hocking, Bijectively related spaces. I.

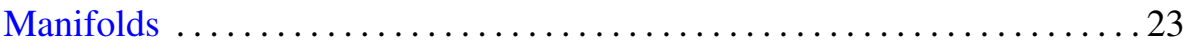

Joel Hass, Complete area minimizing minimal surfaces which are not totally

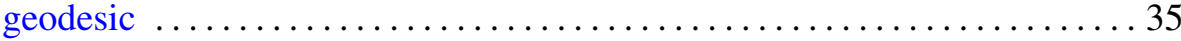

Aarno Hohti, On Ginsburg-Isbell derivatives and ranks of metric spaces .... 39

Richard Howard Hudson, Diophantine determinations of $3^{(p-1) / 8}$ and $5^{(p-1) / 4}$

A. F. Izé and A. Ventura, Asymptotic behavior of a perturbed neutral functional-differential equation related to the solution of the unperturbed

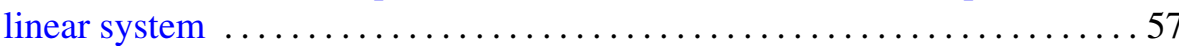

Palle E. T. Jorgensen, Spectral representations of unbounded nonlinear

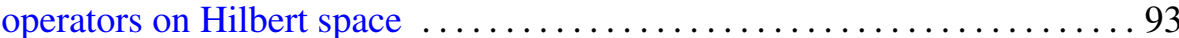

Darrell Conley Kent and Gary Douglas Richardson, Cauchy spaces with

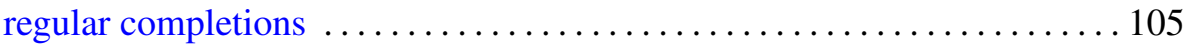

Mark Mahowald, An addendum to: "bo-resolutions" ................ 117

Stuart Wayne Margolis and Jean-Eric Pin, Minimal noncommutative varieties and power varieties

Carla Massaza and Alfio Ragusa, Some conditions on the homology groups of the Koszul complex

Vicente Miquel Molina, Some examples of Riemannian almost-product manifolds

Roderic Murufas, Inverse spectral problems for certain differential operators

Ulrich Oertel, Closed incompressible surfaces in complements of star links

Katsuro Sakai, A characterization of local equiconnectedness

William Victor Smith and Don Harrell Tucker, Weak integral convergence theorems and operator measures 